NBER WORKING PAPER SERIES

\title{
TRADING SPACES: MEDICARE'S REGULATORY SPILLOVERS ON TREATMENT SETTING FOR NON-MEDICARE PATIENTS
}

\author{
Michael Geruso \\ Michael R. Richards \\ Working Paper 28576 \\ http://www.nber.org/papers/w28576
NATIONAL BUREAU OF ECONOMIC RESEARCH
1050 Massachusetts Avenue
Cambridge, MA 02138
March 2021, Revised February 2022

\begin{abstract}
We are grateful to Leila Agha, Lindsey Bullinger, Marika Cabral, Colleen Carey, Alice Chen, Paul Ginsburg, Atul Gupta, Bart Hamilton, Matt Harris, Darius Lakdawalla, Neale Mahoney, Beth Munnich, Maria Polyakova, Rosalie Pacula, David Powell, Seth Richards-Shubik, John Romley, Steve Ryan, Adam Sacarny, Neeraj Sood, and seminar participants at the University of Houston, University of Southern California, and Washington University in St. Louis for many helpful comments on earlier versions of this work. We are also grateful to the participants and organizers of the 2019 ASHEcon meeting, the 2019 Southeastern Health Economics Study Group, and the 2020 Junior Health Economics Summit for opportunities to present this research. We also especially thank Beth Munnich for generous data sharing related to physician ASC investments. We thank the Florida Agency for Health Care Administration (ARCA) for providing the data for this project and note that ARCA was not responsible for any data analyses or interpretations belonging to this work. We have no conflicts of interest to disclose, and all views and errors are our own. The views expressed herein are those of the authors and do not necessarily reflect the views of the National Bureau of Economic Research.
\end{abstract}

At least one co-author has disclosed additional relationships of potential relevance for this research. Further information is available online at http://www.nber.org/papers/w28576.ack

NBER working papers are circulated for discussion and comment purposes. They have not been peer-reviewed or been subject to the review by the NBER Board of Directors that accompanies official NBER publications.

(C) 2021 by Michael Geruso and Michael R. Richards. All rights reserved. Short sections of text, not to exceed two paragraphs, may be quoted without explicit permission provided that full credit, including $\odot$ notice, is given to the source. 
Trading Spaces: Medicare's Regulatory Spillovers on Treatment Setting for Non-Medicare Patients

Michael Geruso and Michael R. Richards

NBER Working Paper No. 28576

March 2021, Revised February 2022

JEL No. H23,H42,H51,I11,I13,I18

\section{ABSTRACT}

Medicare pricing is known to indirectly influence provider prices and care provision for nonMedicare patients; however, Medicare's regulatory externalities beyond fee-setting are less well understood. We study how physicians' outpatient surgery choices for non--Medicare patients responded to Medicare removing a ban on ambulatory surgery center (ASC) use for a specific procedure. Following the rule change, surgeons began reallocating both Medicare and commercially insured patients to ASCs. Specifically, physicians became $70 \%$ more likely to use ASCs for the policy--targeted procedure among their non-Medicare patients. These novel findings demonstrate that Medicare rulemaking affects physician behavior beyond the program's statutory scope.

Michael Geruso

University of Texas at Austin

Department of Economics

1 University Station C3100

Austin, TX 78712

and NBER

mike.geruso@utexas.edu

Michael R. Richards

Baylor University

Department of Economics

One Bear Place \#98003

Waco, TX 76798

michael_richards@baylor.edu 


\section{Introduction}

Medicare covers nearly 60 million individuals and directs roughly a fifth of the United States' more than $\$ 3$ trillion in annual healthcare spending. ${ }^{1}$ Traditional, or fee-for-service (FFS), Medicare enrollment also eclipses that of most other public or private health insurers. Consequently, the Medicare program looms large as a dominant payer in the US healthcare landscape and may influence physician treatment decisions as well as standards of care beyond its patient population and statutory bounds.

Prior research documents important spillover effects from Medicare fee schedules on price negotiations between private insurers and providers as well as on provider behavior toward nonMedicare patient populations. ${ }^{2}$ However, Medicare policy is broader than administrative pricing and often includes care delivery regulations. The impact of these latter policy levers - Medicare's nonprice regulatory decisions - on provider behavior inside and outside of the Medicare market is less well studied. To be clear, by nonprice, we mean Medicare rulemaking that is separate from service fee-setting and includes decisions such as which procedures and equipment are covered for Medicare patients, which facilities or providers can administer certain services, whether providers can balance bill, and so on. Recognizing and measuring externalities from such rules is necessary for formulating optimal Medicare policy and other healthcare regulation. Additionally, studying whether and how Medicare's nonprice regulatory decisions spill over onto physicians' non-Medicare treatment decisions has the potential to shed new light on the formation and

\footnotetext{
${ }^{1}$ These and related statistics from the Centers for Medicare \& Medicaid Services can be found here: https://www.cms.gov/research-statistics-data-and-systems/statistics-trends-andreports/nationalhealthexpenddata/nhe-fact-sheet.html.

${ }^{2}$ More specifically, the former characterizes the linkage between the Medicare fee schedule and negotiated service prices in the commercially insured market-e.g., White (2013), Clemens and Gottlieb (2017), Clemens, Gottlieb, and Molnár (2017), Trish et al. (2017), and Cooper et al. (2019). The latter examines the influence of Medicare payment changes on providers' treatment approaches for the rest of their patient panel-e.g., Sloan, Morrisey, and Valvona (1988), Yip (1998), He and Mellor (2012), and White (2014).
} 
evolution of physician treatment styles, which is an area of longstanding interest (Phelps 2000; Grytten and Sørensen 2003; Epstein and Nicholson 2009; Currie, MacLeod, and Van Parys 2016; Molitor 2018; Cutler et al. 2019) —including how a given physician's treatment style converges or diverges across patients with different insurance (Newhouse and Marquis 1978; Glied and Graff Zivin 2002; Frank and Zeckhauser 2007).

In this paper, we investigate Medicare's regulatory externalities on physicians' medical decision-making for their non-Medicare (i.e., commercially insured) patients. In January 2008, Medicare reversed a prior regulatory stance that banned a specific surgical procedure from taking place within an ambulatory surgery center (ASC) for FFS Medicare patients. ASCs, also known as outpatient surgery centers or same-day surgery centers, are stand-alone, non-hospital facilities that are typically lower-cost treatment setting options for procedures not requiring an inpatient stay. Prior to 2008, Medicare would only pay for laparoscopic cholecystectomy surgeries (i.e., minimally invasive gallbladder removals) if these were administered within a hospital outpatient or inpatient department—effectively prohibiting Medicare cases within ASC settings.

The 2008 policy change by Medicare provides an ideal quasi-experiment to investigate spillovers from Medicare's nonprice regulation. As we describe below, the roll-back of Medicare's ban was purely an administrative change. It was not a response to changes in medical technology, perceived patient clinical benefits, or physician preferences. We also show that it did not coincide with the relaxing of other Medicare treatment setting restrictions for other procedures important to the affected surgeons. Instead, the original Medicare ban existed without a clear evidence-base and did not necessarily align with care delivery parameters stipulated by other payers or similar surgical services. The narrow application of the ban (laparoscopic cholecystectomy surgeries) also meant that only surgeons performing this specific operation were plausibly affected by its removal, 
which generates well-defined treatment and control physician groups. These features of the quasiexperiment, along with the sharp timing of the deregulation event, are useful in disentangling policy effects from underlying trends and allow us to ask whether a change to the permissible service delivery setting for Medicare patients ultimately impacts physicians' choice of setting for non-Medicare, commercially insured patients receiving the same procedure.

A key institutional feature for our outpatient surgery context is that, when permitted by the relevant payer, physicians often divide their patients receiving the same surgical service between hospital outpatient department (HOPD) and ASC delivery settings, rather than operate solely within one. This behavior has been of economic interest, and David and Neuman (2011) usefully refers to such physicians as "splitters" since they split their relevant case load across facility types. David and Neuman (2011), along with Yee (2011) and Munnich and Parente (2018), examine what drives physician splitters to allocate the marginal patient to a particular facility type. Yet, surprisingly little economics research to date has examined the underlying determinants of a physician choosing to split a given procedure between ASC and HOPD options in the first place. ${ }^{3}$ In what follows, we demonstrate that Medicare regulation can have an immediate impact on physicians' decisions to split outpatient surgeries for Medicare and non-Medicare cases alike.

To build on this literature, we analyze physicians' decisions to split surgeries across settings as our primary outcome of interest. As we describe in more detail below, there are several reasons to expect that a physician's decision to begin using ASCs for some of her Medicare patients might be jointly made with her decision to begin using ASCs for some patients with other insurance coverage. To motivate this possibility, we note an empirical regularity: a physician's splitter status for her Medicare outpatient procedures is highly correlated with her splitter status for the same

\footnotetext{
${ }^{3}$ The lone exception that we are aware of is a contemporaneous paper (Munnich et al., 2021) that shows a sharp and substantive ASC extensive margin effect when physicians newly acquire financial stakes in one or more ASCs.
} 
procedures paid for by commercial insurers. In our data (described below) and across all unique physician-procedure combinations in 2007, we observe that conditional on a physician behaving as a splitter for a given Medicare procedure, the probability that the same physician also divides her corresponding commercial procedure volume between HOPDs and ASCs is high ( $88 \%$, see Figure 1). ${ }^{4}$ Conditional on a physician not splitting a given Medicare procedure between settings, the probability that she nonetheless does so for the same procedure reimbursed by commercial insurers is low (16\%). In principle, this within-physician, cross-payer correlation in surgical practice style could entirely reflect physician and patient preferences or the underlying complexity of the procedures with no feedback or spillovers to or from the Medicare program. Yet, in this paper, we show that this is not the case. We find that Medicare rules have a large causal impact on the care delivery setting chosen for non-Medicare patients, holding physicians and procedures fixed.

We establish the spillover effects of Medicare's deregulation change in the universe of outpatient procedure discharge records from Florida from 2005 through 2011. We first show a sharp uptick in the likelihood of splitting FFS Medicare laparoscopic cholecystectomy surgeries once Medicare removes the ASC ban for these surgeries. ${ }^{5}$ The impact is clearly visible in a simple difference-in-differences plot that compares surgeons who perform the procedure to other

\footnotetext{
${ }^{4}$ Authors' cross-sectional calculation from the universe of outpatient procedure records in Florida during 2007. Full data details are provided in Section 3. 34\% of commercial outpatient procedures are split between both HOPD and ASC settings in the 2007 data. This subset of outpatient procedures is most likely to be comprised of procedures (e.g., surgeries) that can be safely performed in an ASC for lower risk patients but not for patients with higher risk factors for complications and/or expected greater operative and post-operative resource needs. Appendix Figure B1 includes an intensive margin comparison across payers and shows a strong correspondence.

${ }^{5}$ Throughout, we use Medicare to denote fee-for-service (FFS) Medicare. The Medicare Advantage (MA) market is also of interest. However, the volume of relevant procedures for MA enrollees in the early years of our data is low. In our sample in 2005, Commercial Insurers (non-MA) covered $66.0 \%$ of LC procedures, Traditional Medicare covered 17.4\%, Medicaid covered 6.7\%, and all Medicare Advantage insurers combined covered just 3.5\%.
} 
physicians over the relevant timeframe. This tendency to begin splitting the targeted Medicare surgeries between outpatient delivery settings (i.e., HOPDs and ASCs) is a direct and expected first-order policy effect and is consistent with physicians reoptimizing their choice of setting for Medicare patients after Medicare permits a new procedure-location combination.

After establishing the direct policy effect, we investigate our spillover effect of interest: physician-level splitter status for non-Medicare patients receiving the same surgical procedure. By comparing surgeons who perform the targeted procedure in Medicare to all other physicians in a difference-in-differences framework, we show a $25 \%$ increase over pre-policy levels in the probability a physician splits her commercially insured laparoscopic cholecystectomy surgeries during the first year of the Medicare rule change. The effect grows and stabilizes to a $70 \%$ increase by the third post-policy year and thus reveals that the Medicare rule change had a substantial impact on how physicians provided care to non-Medicare patients.

Thus, our main contribution is to establish, for the first time, that Medicare regulation prohibiting certain modes of service provision substantially affects physician practice patterns for non-Medicare patient populations. The physician behavior changes we document have important implications beyond the narrow surgical procedure that we study. The same type of Medicare regulatory policy is in place (statically) for essentially every surgical procedure in US healthcare delivery - either prohibiting or permitting certain modes and places of care. These same rules are also an active source of contemporary debate and policy shifts. ${ }^{6}$ In this way, our identifying

\footnotetext{
${ }^{6}$ Examples of industry commentaries on policy alignments and contrasts across the two recent executive branch administrations can be found here: https://www.beckersasc.com/asc-news/trump-vs-biden-who-is-better-forascs.html and here:

https://www.ascassociation.org/asca/aboutus/latestnews/newsarchive/newsarchive2021/july2021/202107medicare20 22 proposedpaymentrule. The Centers for Medicare and Medicaid Services (CMS) is currently proposing to reinstate some previous prohibitions on ASC-delivered surgeries (see here https://www.cms.gov/newsroom/fact-sheets/cy2022-medicare-hospital-outpatient-prospective-payment-system-and-ambulatory-surgical-center).
} 
variation offers a rare opportunity to understand the broader impacts of this type of Medicare administrative decision-making, which can inform ongoing deliberations on related policies tied to a wider variety of medical services.

Our findings also contribute to an active literature on the externalities from public health insurance programs for the healthcare economy. This includes work on public insurance payments influencing private insurer payments (e.g., White (2013), Clemens and Gottlieb (2017), Clemens, Gottlieb, and Molnár (2017), Trish et al. (2017), and Cooper et al. (2019)), public insurance payments influencing physician behavior toward other patient groups (Sloan, Morrisey, and Valvona (1988), Yip (1998), He and Mellor (2012), White (2014), Meyers et al. (2019), Einav et al. (2020), Wilcock et al. (2020), and Chen et al. (2022)), and public payer reimbursements influencing drug development (Yurukoglu et al. 2017), among other outcomes. In contrast with prior work that has largely focused on administrative pricing, ${ }^{7}$ we provide novel evidence of Medicare's own- and cross-market effects from nonprice regulation. We also view our results as complementing a long literature on physician behavior and decision-making in a multi-payer environment (McGuire and Pauly 1991). ${ }^{8}$ Extending this prior literature, our findings indicate that the regulatory environment interacts with physician decision-making even when the regulations do not directly bind. Our work consequently reveals the long reach of Medicare rulemaking and

\footnotetext{
${ }^{7}$ Barnett, Olenski, and Sacarny (2020) is a notable exception. This recent study offers evidence on non-price externalities by showing how letters from Medicare warning physicians against overuse of antipsychotic drugs also reduced prescribing to privately insured patients for these same drugs.

${ }^{8}$ Related empirical works include within-Medicare spillovers from greater Medicare Advantage penetration (Chernew, DeCicca, and Town 2008; Baicker, Chernew, and Robbins 2013; Baicker and Robbins 2015; Callison 2016) as well as studies of public and private payer demand shocks within the mixed economy for physician services (Garthwaite 2012; Bond and White 2013; Joynt et al. 2013, 2015; He, McInerney, and Mellor 2015; McInerney, Mellor, and Sabik 2017; Glied and Hong 2018; Richards and Tello-Trillo 2019; Carey, Miller, and Wherry 2020). Some of this research has documented physician tendencies toward similar treatment styles for patients, regardless of payer (Glied and Graff Zivin 2002), as well as the use of behavioral heuristics and norm following in physician decision-making (Frank and Zeckhauser 2007).
} 
its ability to shape physician behavior and healthcare delivery beyond the statutory scope of the regulation.

\section{Background}

\subsection{Physicians and outpatient surgery care}

Absent a regulatory constraint, ASCs directly compete with hospitals for a variety of outpatient procedures (Bian and Morrisey 2007; Courtemanche and Plotzke 2010; Carey, Burgess, and Young 2011; Carey 2017; Whaley and Brown 2018; Baker, Bundorf, and Kessler 2019). Hospitals are medically appropriate for higher risk patients and procedures, but physicians may prefer using ASCs for at least some patients and procedures. ${ }^{9}$

Intuitively, the complexity - and hence adverse event risks - accompanying a procedure are likely to influence physicians' willingness to make use of both treatment settings. For example, splitting is likely to be rarer for low complexity outpatient procedures (e.g., diagnostic tests like colonoscopies or endoscopies), for which there may be little or no need for the higher intensity and higher resourced HOPD facilities. Likewise, physicians may be less inclined to rely on ASCs at all for certain high-complexity procedures that carry greater risks and are therefore best suited for hospital-based delivery. However, for surgeries of moderate complexity (such as laparoscopic cholecystectomies), a subset of patients is likely to be medically appropriate for the lower intensity ASC setting - making the choice to utilize both treatment settings (i.e., split cases) a key physician decision-making margin for such procedures.

\footnotetext{
${ }^{9}$ Empirical research also supports that ASCs offer equivalent care to HOPDs that is often more convenient and lower cost for patients (Paquette, Smink, and Finlayson 2008; Grisel et al. 2009; Munnich and Parente 2014; Weber 2014; Carey 2015; Munnich and Parente 2018; Aouad, Brown, and Whaley 2019; Sood and Whaley 2019), which gives physicians' choice of setting welfare implications for patients as well as earnings implications for these rival outpatient facilities.
} 
The factors that lead physicians to schedule some of their patients at ASCs for a given outpatient surgery are also likely to be common across payers, within the same physician. For example, the ASC might provide physician amenities (such as specialized staffing and administrative support services) as well as preferential scheduling. Physicians may also have heterogeneous beliefs about or concern for how the treatment setting impacts patient convenience and clinical outcomes. Financial incentives could apply as well, at least for the small minority of physicians with direct ownership stakes in the ASC industry (Lynk and Longley 2002; David and Neuman 2011; Plotzke and Courtemanche 2011; Dyrda 2017; Munnich et al. 2021). ${ }^{10}$ Such considerations, along with other idiosyncratic physician factors, might drive a cross-sectional correlation between ASC use for a given procedure type inside and outside of Medicare (as in Figure 1) without any causal spillover from Medicare policy. Yet, other factors (described next) would imply a joint decision process for physicians, across their Medicare and non-Medicare patients—and hence the possibility of cross-payer spillovers.

\subsection{Potential for cross-payer spillovers in treatment setting}

In order to guide our empirical investigations, it is useful to first consider how physicians decide whether to perform a given procedure at an ASC for at least some of their patients, with exclusive use of the HOPD setting serving as the outside option. Surgeons are typically multiproduct firms that supply a variety of services (i.e., procedures) to patients. These surgeons also tend to sell the same service to multiple payers—e.g., Medicare, Medicare Advantage, Medicaid, and non-elderly commercial (private) insurers.

\footnotetext{
${ }^{10}$ Note, reimbursements for physicians (i.e., the professional component of payment) is independent of the chosen treatment setting. Thus, even physician ASC equity owners are only indirectly rewarded for choosing an ASC instead of HOPD via their partial claim on the facility payment, which is separate from the physician payment.
} 
For a sufficiently complex procedure, such as a laparoscopic cholecystectomy (LC), some patients will always receive care in a HOPD setting. A physician's decision to use an ASC for some other patients is equivalent to the decision to divide her caseload across the two treatment settings. The choice to perform a procedure at an ASC for at least some of her patients (i.e., behave as a "splitter") depends on physician preferences and patient characteristics. ${ }^{11}$

Importantly, the decision to split cases across treatment settings may also depend on the physician's use of ASCs for other payer-procedure combinations. Even holding fixed physician and patient preferences as well as reimbursement, spillovers across procedure types and/or payers could occur for reasons of physician convenience. For example, opportunities to block-schedule patients within the same facility (i.e., perform Medicare and non-Medicare surgeries back-to-back) could reduce travel between facilities and perhaps promote more efficient care delivery (e.g., minimize operating room turnover time between patients and/or facilitate better surgical team performance through consistent staffing over the course of the day). Medicare loosening restrictions on certain procedures at ASCs could also cause physicians to be willing to perform these same procedures for the commercially insured at ASCs through a litigation risk channel. Specifically, in medical malpractice litigation, the standard of care depends on what other providers in the physician's geographic area are doing for similar patients. A Medicare rule change that induces more Medicare patients to be treated at ASCs would change the prevailing treatment norms and therefore the potential legal exposure associated with performing an identical surgery for non-Medicare patients in the same setting. ${ }^{12}$

\footnotetext{
${ }^{11}$ As we model formally in Appendix A, it may depend upon a variety of factors, such as the reimbursement rule for the payer-procedure combination, the appropriateness for ASC use among the physician's patients, the physician's beliefs about patient preferences, and idiosyncratic physician preferences.

${ }^{12}$ In the absence of such considerations, one could view the regulatory change as merely a price change from zero to a positive amount, rather than removal of a ban. However, even if the Medicare 'ban' we study were viewed purely as a facility price change (from $\$ 0$ to about $\$ 1,900$ ), the change is large compared to prior studies that have
} 
Alternatively, or in parallel with effects operating via physician convenience, commercial insurers could voluntarily adopt the regulatory stance of Medicare. In our context, this type of "rule following" would involve commercial insurers disallowing reimbursement for the relevant procedure at ASCs before 2008 and then allowing it after 2008. Complementarities and convenience explanations rely on physician agency; however, rule following would be under the control of private insurers. In either case or in combination, it is plausible that physicians would consider both payer types (i.e., Medicare and non-Medicare) when deciding to use the ASC option for either.

\subsection{Medicare's 2008 rule change}

Medicare has reimbursed physicians and facilities for procedures performed within ASCs for over three decades. More than 5,000 ASCs are currently operational and Medicare certified across the US, and the ASC industry has collected over $\$ 4$ billion annually in Medicare revenue in recent years (MedPAC 2019). ${ }^{13}$ The associated fee schedule has evolved at different times—see He and Mellor $(2012,2013)$ and Munnich and Parente (2018) for detailed histories-but importantly, Medicare patients and their physicians are accustomed to care delivered within ASC settings. However, not all procedures for Medicare patients are allowed to take place within an ASC, which provides an opportunity to exploit a targeted Medicare rule change concerning ASCs for our identification strategy.

identified effects of Medicare price changes, where such changes are typically on the order of $10 \%$ or less. (e.g., Clemens and Gottlieb, 2014; Clemens and Gottlieb, 2017; Clemens, Gottlieb, and Molnar 2017).

${ }^{13}$ In 2017 specifically, Medicare made $\$ 4.6$ billion in payments to ASCs (MedPAC 2019). For reference, in that same year, Medicare spent $\$ 286.5$ billion on hospital payments and $\$ 37.4$ billion on nursing care facilities and retirement communities (CMS, Office of the Actuary, National Health Statistics Group). 
Effective January 12008 , Medicare amended its rules to allow LC surgeries to be performed at ASCs for Medicare FFS patients for the first time. ${ }^{14}$ The decision followed a Government Accountability Office (GAO) study of ASCs mandated by the Medicare Modernization Act (MMA) of 2003 that was completed in November 2006 and laid the basis for the subsequent reforms to the Medicare ASC facility fee schedule. ${ }^{15}$

Importantly, the pre-2008 refusal to reimburse for this specific procedure performed within an ASC is typically characterized as an administrative oversight, rather than a prudent concern for beneficiaries' safety (Meredith 2008; OR Manager 2007). ${ }^{16}$ For example, among non-Medicare patient populations, LC surgeries performed within ASCs had been shown to be equally safe and successful as HOPDs and were associated with lower charges (Paquette et al. 2008). In short, the nature and timing of this nationwide policy change was plausibly the result of a GAO study

\footnotetext{
${ }^{14}$ Recall, this surgery is a minimally invasive gallbladder removal. The laparoscopic cholecystectomy technique globally entered mainstream medicine in the mid- to late-1980s and soon became the norm. Its popularity stems from its many advantages over open cholecystectomy surgery, including less postoperative pain, shorter hospital stays, and more rapid recovery. The development and spread of the laparoscopic cholecystectomy technique has even been described as a watershed moment for minimally invasive surgery applied to many other human organs (Polychronidis et al. 2008).

${ }^{15}$ Because the finalized rule change was published in the Federal Register in November 2007, it is possible that spillovers beyond Medicare could have occurred several months prior to the rule change - though in Medicare itself, the ban remained in effect until January 1, 2008. The surgical trade press also highlighted the rule change in late 2007 (e.g., OR Manager (2007)) and soon after its arrival (e.g., Meredith (2008)). Additionally, at least in principle, the anticipatory responses could have occurred as early as the publication of the GAO study recommending a change in November 2006, 14 months prior to implementation, though there would be significant uncertainty as to the CMS rulemaking response to the GAO report at that stage. It is also possible that physician adjustments would occur with a lag, if transitioning to a new care setting required new relationships with ASCs and/or modifying their work schedules. Our empirical strategy (Section 3.2) allows us to explore these potentially important dynamics in physician behavior change. Specifically, we directly analyze the time pattern of responses to shed light on anticipatory responses as well as any lag in the physician response - not only in a difference-in-differences specification but in pure (time) event-studies focusing on the treated group of LC surgeons. Also, detailed historical reimbursement information can be found here: https://www.cms.gov/Medicare/Medicare-Fee-for-ServicePayment/ASCPayment/downloads/ASC_QAs_03072008.pdf as well as here: https://www.cms.gov/Regulationsand-Guidance/Regulations-and-Policies/QuarterlyProviderUpdates/downloads/cms1392fc.pdf
}

\footnotetext{
${ }^{16}$ For example, the 2008 decision was not based on recent clinical trial data or technological breakthrough
} pertaining to the LC technique within ASC settings. 
(mandated by Congressional action), rather than changes in the underlying technology, medical science, or physician and patient preferences.

Table 1 briefly describes the case characteristics for this specific surgery, compared to all other outpatient procedures in our data. Across payer types (i.e., commercially insured and traditional Medicare), LC surgery patients tend to be younger, on average, and are more likely to be female. This specific surgery is also more common among non-Medicare populations, with commercial cases outnumbering Medicare cases in our data by $250 \% .{ }^{17}$ We also note that while commercial insurers may adopt Medicare regulatory positions, they are not required to. In fact, we observe commercially insured laparoscopic cholecystectomy surgeries taking place within ASCs prior to 2008. Additionally, within Appendix Figure B2, we see no evidence of a market expansion effect of the rule change for any payer group. While the policy may influence where an LC takes place, it does not seem to impact the quantity of LCs.

\subsection{Empirical implications and interpretations}

Given the plausible scenarios described in Section 2.2, the narrow Medicare regulatory change described in Section 2.3 targeting LCs could potentially influence physicians' treatment setting decisions for three broad categories of payer-procedure combinations: 1) non-Medicare LCs 2) Medicare non-LCs and/or 3) non-Medicare non-LCs. Guided by these considerations, in what follows we consider all LC physicians as treated in the post-period, including when we examine their non-LC procedures. We use physicians who are not exposed to the regulatory change (i.e., who never perform the Medicare policy-targeted LC surgery) as a control group for measuring the

\footnotetext{
${ }^{17}$ Authors' calculations based on the universe of ambulatory procedure data from Florida (fully described within Section 3).
} 
size of spillovers. We also allow for the possibility of heterogenous impacts across treated physicians, who may differ in their potential adjustment costs and/or financial interests linked to the ASC industry.

Observing a change in the use of ASCs for non-targeted procedures could be consistent with a variety of spillover mechanisms, as noted above. Rule following is the key insurer-centered explanation; however, physician-centered explanations include the possibility that the physician's cost of ASC scheduling is falling in the variety of procedures available to be performed at ASCs and/or a "norms hypothesis" for physician behavior (Newhouse and Marquis 1978; Glied and Graff Zivin 2002; Frank and Zeckhauser 2007). ${ }^{18}$ Ultimately, our paper aims to empirically establish whether cross-payer influences exist, not to identify the particular channel—which our data are not well-suited for (discussed in Section 6).

\section{Empirical Strategy for Own and Spillover Medicare Deregulation Effects}

\subsection{Data}

Our set of analyses leverages the universe of ambulatory (i.e., outpatient) procedure discharge records from the state of Florida, which we obtained from the Florida Agency for Health Care Administration (AHCA). Florida is home to a large share of the nation's Medicare population-3 to 4 million beneficiaries in recent years, which is second only to California.

Although discharge records lack the kind of reimbursement information typically found in claims data-meaning we cannot observe whether and how commercial reimbursement amounts

\footnotetext{
${ }^{18}$ This idea argues that tangible and cognitive costs can lead physicians to adopt similar treatment styles for different patients-i.e., limit treatment customization for a given medical problem (Newhouse and Marquis 1978; Glied and Graff Zivin 2002; Frank and Zeckhauser 2007). Applied to our context, forces related to physician norms or coordination costs do not affect the choice of treatment (i.e., the procedure to be performed) but instead influence where treatment takes place.
} 
evolve following the Medicare rule change—-the discharge records possess the key advantage for our purposes of complete and consistent physician longitudinal identifiers across records, payers, and treatment settings. Specifically, the discharge records clearly indicate whether a given ambulatory case was performed within a HOPD or ASC setting and contain the Florida medical license belonging to the physician performing the case. ${ }^{19}$ These features, coupled with the ability to observe discharges associated with all payers within the state, allow us to completely track physicians' allocations of procedures across these two treatment settings for our two payer groups of interest: traditional Medicare and the (non-Medicare) commercially insured. ${ }^{20}$ Having consistent physician identifiers over time and across public and private payers is critical to addressing our research question. ${ }^{21}$

Our administrative data span the first quarter of 2005 through the fourth quarter of 2011, which consequently captures ambulatory procedures several years before the Medicare rule change as well as several years after the policy's debut. These detailed records include a rich set of variables, such as patient demographic information and associated diagnosis and procedure codes. Procedure codes appear in the discharge data as Current Procedure Terminology (CPT) or Health Care Common Procedure Coding System (HCPCS) codes.

\footnotetext{
${ }^{19} \mathrm{We}$ ignore cases belonging to highly specialized points of care (e.g., lithotripsy centers and cardiac catheterization centers) that are not relevant to our analytic context and research questions. These cases also account for less than $1 \%$ of discharges in a typical year.

${ }^{20}$ These are the two payers that makeup the overwhelming majority of outpatient procedure cases, especially for ASCs in our data. Other specific payers (e.g., Medicaid) are vanishingly small components of the typical Florida ASC's payer mix. We also note that nationally more than $80 \%$ of ambulatory (outpatient) surgeries are estimated to have either commercial insurance or Medicare as the main payer (Hall et al. 2017).

${ }^{21}$ The discharge data we use are ideal given that no all-payer claims database exists that covers our period of study (2005-2011) and contains consistent physician identifiers over time. Having this latter element as well as having it available across public and private payers are critical to tracking the physician response that we study.
} 
In what follows, we exploit these data features to construct a time series of physician behavior that reflects the use of ASC and HOPD treatment settings. In most cases, the unit of analysis is the physician by procedure by payer by time period.

\subsection{Estimation Procedure}

We begin by estimating the first-order (direct) effect of the Medicare ASC regulatory policy change. This is both an important policy response to document in itself and a natural precondition for certain types of spillover channels from Medicare onto commercial patients-e.g., via convenience complementarities, capacity-constraint-driven crowd out, or insurer rule-following. We then use an analogous approach (fully described below) to estimate the indirect effect of the Medicare deregulation change on treatment setting choices for commercially insured patients.

When estimating the policy's direct and indirect effects, we rely on a difference-indifferences (DD) design. The narrowly targeted nature of the Medicare rule change lends itself to a well-defined treatment versus control group setup. Specifically, we define the treatment group as LC surgeons - that is, surgeons who perform LC procedures. ${ }^{22}$ We classify a physician as an LC surgeon if she performs at least one LC case for the relevant payer type (i.e., Medicare or commercial insurance) within each half year. Among LC surgeons, the share of outpatient procedures made up of LC surgeries is typically around 10\% - making it one of the more common medical services performed by this group. ${ }^{23}$ We aggregate the quarterly data to the half-year level

\footnotetext{
${ }^{22}$ Recall, we are using the 'LC procedures' and 'LC surgeries' abbreviations as convenient shorthand for laparoscopic cholecystectomy surgeries. We maintain this abbreviation in Sections 4 and 5.

${ }^{23}$ The typical LC surgeon is trained as a "general surgeon" during the required post-medical school residency program; therefore, the surgeon will typically not exclusively perform LC surgeries. For example, among our analytic sample of Medicare LC surgeons used to produce the DD results in Section 4, they performed over 700 unique outpatient procedures (defined as unique CPT codes) for Florida Medicare beneficiaries in 2007.
} 
because procedures could have few or no cases for a given physician-payer combination in a given quarter.

We observe a balanced panel of 387 unique LC surgeons for the commercial market analyses that are observed performing LCs in each of our 14 half-year periods spanning our 20052011 time frame. ${ }^{24} \mathrm{We}$ consider this set of surgeons to be our primary physicians of interest for detecting Medicare rulemaking externalities (spillovers). Recall that the Medicare LC market is considerably smaller than the commercial market (Table 1). Consequently, of these 387 LC surgeons of interest, not all of them have positive Medicare LC case volumes in every half-year; however, over $70 \%$ have Medicare LCs in at least 12 of our analytic time periods, and $90 \%$ have Medicare LCs in at least 8 time periods. We therefore define two alternative treatment group samples for our Medicare (direct effect) analyses. The first is unrestrictive (i.e., an unbalanced panel) and requires only a single Medicare LC surgery to be included in the treatment group. This sample includes 386 of the original 387 commercial LC surgeons. The second sample definition for Medicare LC surgeons restricts to the subset of Medicare LC surgeons that have a balanced panel of Medicare cases (i.e., Medicare LC surgeries observed in every period), yielding 162 LC surgeons. Generating estimates from both samples allows us to ensure that our findings are not sensitive to these sample inclusion criteria.

We are interested in whether the Medicare policy change causes some physicians to begin using both treatment setting options for the relevant procedure. We define a surgeon-proceduretime period-payer observation as having the "splitter" treatment style in a given half-year if the surgeon divides her patients for that procedure between the ASC and HOPD settings for that payer

\footnotetext{
${ }^{24}$ Balance here means that the LC surgeon is performing LC procedures in every single half year increment of the sample period. Physicians inconsistently performing LC procedures are dropped from the commercial analytic sample.
} 
in that half-year, which is consistent with the David and Neuman (2011) terminology. The variable, which is the focal outcome examined in the regressions below, is therefore binary in its construction and is virtually synonymous with extensive margin ASC use for this specific surgery among our treatment group physicians. $^{25}$

We verified that the policy change we exploit was not part of a wave of new ASC permissions affecting other common procedure types that could confound our identification strategy. In particular, we examined all procedure (CPT) codes belonging to our treatment group physicians and their Medicare patient population in 2007, regardless of the procedure setting. Of the more than 700 such codes, the top 50 ranked by volume accounted for $77 \%$ of the overall Medicare outpatient procedure volume for these physicians. We manually reviewed the publiclyposted Medicare facility reimbursement policy for ASCs for each of these top-50 codes. Of these, only LCs were a primary procedure that was both not eligible for Medicare reimbursement if performed in an ASC prior to 2008 and newly eligible for Medicare reimbursement in $2008 .^{26}$

To provide our DD control group, we rely on non-LC physicians - that is, physicians who never perform the policy-targeted procedure at any point for any payer in our data. Importantly, it would be inappropriate to include the non-LC procedures of treatment group physicians in our control group because of the arguments made in Section 2, which suggest that the Medicare policy

\footnotetext{
${ }^{25}$ Only a few (and very low volume) LC surgeons transition from $0 \%$ to $100 \%$ ASC use for LC patients. Although "splitter" is virtually synonymous with extensive margin ASC use for the LC procedure, other (simpler, low-risk) procedures, such as spinal injections and colonoscopies, may be performed exclusively in ASCs by a given physician.

${ }^{26}$ Four of the top 50 codes newly included a Medicare ASC facility payment in 2008; however, all four were add-on codes (i.e., the application of a boot following an ankle or foot surgery or the use of a special surgical closure technique), rather than primary procedure codes, and generally had a fee level of under $\$ 30$. We also note that there was no contemporaneous variation in the physician reimbursement that would have made ASCs more attractive for performing LCs. Recall for our LC procedure of interest—and indeed, for all outpatient procedures-Medicare does not reimburse the physician differently as a function of where the surgery took place. The physician professional fee is the same in the ASC and HOPD settings.
} 
targeting LCs could affect how LC physicians organize care for their other procedures. We explicitly examine this possibility in Section 5, but for our primary analysis in Section 4, we exclude observations for the non-LC procedures belonging to LC physicians. We then use all possible remaining physician-procedure combinations within a payer (i.e., Medicare or commercially insured) to construct analogous time series tuples (physician $\times$ procedure $\times$ payer $\times$ period). Including all procedures by all non-LC physicians is a natural way to form the control group, given the targeted nature of the Medicare policy change, and has the added benefit of avoiding any arbitrary selection of a specific procedure to serve as the comparison group.

To classify what constitutes a control procedure, we include only the "principal CPT code" (i.e., the primary service the patient is receiving as an outpatient procedure) within the discharge records. ${ }^{27}$ This step avoids including in our estimation any ancillary procedures (e.g., blood tests, imaging tests, or transfusions) that are merely incident to the main outpatient services delivered to patients. Because observations are at the physician $\times$ procedure $\times$ payer $\times$ period level, a control group physician may comprise multiple physician-procedure tuples, if they consistently supply more than one unique procedure for the relevant payer over the 2005 to 2011 period. This is likely since most physicians are multiproduct firms for a given payer. We end up with roughly 7,000 unique physician-procedure combinations $(q)$ within our control group for our analysis of the direct effects within Medicare and approximately 11,000 unique combinations for our analysis of spillover effects on commercially insured patients.

We first estimate a simple pre-/post- DD equation separately for our two main analytic samples (i.e., the Medicare and commercial markets):

\footnotetext{
${ }^{27}$ The set of principle CPT code candidates is drawn from the 2007 discharge data. The principal CPT is also a distinct data field in the discharge records (i.e., it is separate from additional CPT code variable columns, which can be used for recording ancillary services incident to the principal procedure performed during the encounter).
} 


$$
\text { Splitter }_{q t}=\delta\left(1\left(\text { Post }_{t}\right) \times 1\left(\text { LCSurgeon }_{q}\right)\right)+\theta_{q}+\eta_{t}+\varepsilon_{q t}
$$

Splitter is defined as above. The Post variable is equal to one for all half-years after and including the beginning of 2008 (when Medicare removes the ASC ban for LC surgeries). LCSurgeon is time-invariant and equal to one for LC surgeons - those directly exposed to the Medicare policy change. We also include a full vector of physician-by-procedure fixed effects $(\theta)$ and time period fixed effects $(\eta)$ for our full study period (2005-2011), which subsume the LCSurgeon main effect and Post main effect, respectively. ${ }^{28}$ The $\delta$ parameter identifies the pooled post-period DD estimate, which summarizes the overall post-period effect from the policy change.

To enhance the flexibility of the model and to allow for examination of pre-trends, we adapt our DD analytic setup to an event study difference-in-differences framework:

$$
\text { Splitter }_{q t}=\sum_{n=-6}^{7} \delta_{n}\left(1\left(\text { LCSurgeon }_{q}\right) \times 1(t+n)\right)+\theta_{q}+\eta_{t}+\varepsilon_{q t}
$$

Equation (2) includes fixed effects for each physician-by-procedure and for each half-year time period as in Equation (1). The estimating equation also interacts the vector of time period indicators with our LCSurgeon variable to produce a series of $\left(\delta_{n}\right)$ coefficients. The omitted, reference time point is the second half of $2007(t-1)$, which immediately precedes the Medicare regulatory change. The $\left[\delta_{-6}-\delta_{-1}\right]$ coefficients allow us to examine the pre-policy trends of LC surgeons relative to the control group. Non-parallel differential pre-trends would suggest a possible violation

\footnotetext{
${ }^{28}$ Note, for the treatment group (LC surgeons), it is ultimately a physician fixed effect since all of their other (nonLC) procedure activity is deliberately excluded from the analytic sample.
} 
of the identifying assumption that the treatment and control group outcomes would have evolved similarly in the absence of the regulatory shock to the treatment group. The $\left[\delta_{0}-\delta_{7}\right]$ coefficients flexibly allow for dynamics in the policy response over the subsequent 4-year period for the direct (Medicare) and indirect (commercial) analyses. Finally, our standard errors are clustered at the physician level throughout the main DD and sensitivity analyses (described in Section 4.2). ${ }^{29}$

\section{Results for Own and Spillover Deregulation Effects}

\subsection{Main Results}

We begin with the DD results for Medicare's policy effect on care delivery for its own enrollees, where the post-policy differential effect is summarized in a single coefficient (see Equation (1) in Section 3). In column 1 of Table 2, we identify a large and highly significant 8.8-percentage point uptick in the likelihood of surgeons behaving as a splitter for their Medicare LC patients. This DD result is the direct effect of the policy change whereby Medicare rulemaking affects the provision of the Medicare-reimbursed service targeted by the regulation (in the expected direction).

Column 2 of Table 2 and Figure 2 display the corresponding event study results using Equation (2), which more flexibly fits the data and allows for examination of pre-trends. For the Medicare (direct) effect of deregulation, the pre-policy coefficients are close to zero in magnitude and never statistically different from it, with the exception of the first half of 2005. This is consistent with the pre-2008 Medicare policy amounting to a ban on LCs being performed for its beneficiaries in the ASC setting. With the introduction of the Medicare rule change, however, there

\footnotetext{
${ }^{29}$ For completeness, we also checked if clustering at the physician-procedure level had a material impact on our standard error sizes for our direct and indirect policy effect estimates - e.g., if uneven cluster group sizes when clustering at the physician level led to an underestimation of our standard errors and hence over-rejection of the null (e.g., see MacKinnon and Webb 2017). The statistical significance of our findings is entirely robust to the alternative clustering.
} 
is a sharp and precisely estimated 5-6 percentage point uptick in the probability that LC surgeons splitting their Medicare LC patients across treatment settings within the first post-policy year (i.e., time $t$ and $t+1))^{30}$ The policy response grows throughout the post-implementation period, with the effect size nearly twice as large (i.e., a roughly $12-13$ percentage point differential increase) at its peak when compared to the initial year after the deregulation intervention. We therefore interpret the DD result from Table 2 and the corresponding event study results (Table 2 and Figure 2) as offering strong evidence of a direct and presumably intended policy effect: surgeons exposed to the deregulatory change took advantage of the new delivery setting opportunity after the Medicare administrators approved the use of ASC treatment settings.

We now turn to spillover effects. Recall that the samples used in this spillover analysis include only observations for procedures paid for in the non-Medicare (and non-Medicaid) commercially insured market. The treatment group is composed of all LC surgeons consistently observed over our 2005-2011 period. Their non-LC procedures are omitted (and investigated separately below). The control group includes the commercial market procedure $\times$ physician observations for physicians never performing an LC surgery. Note that there can be no "direct" effect of the policy in the spillover sample of LC surgeons because there is no statutory connection between Medicare's payment policy and the treatment setting choices for non-Medicare patients.

Within column 1 of Table 3, we find a 6.5-percentage point increase in the likelihood of LC physicians behaving as a splitter for their commercial payer LC patients-indicative of a spillover effect from the Medicare policy change. The estimate is also precise and translates to a $52 \%$ increase over the pre-period propensity to use both surgical setting options for these patients.

\footnotetext{
${ }^{30}$ Recall, this difference-in-differences effect is relative to any changes in splitter status among non-LC physicians performing non-LC Medicare procedures.
} 
Column 2 of Table 3 and Figure 3 display the corresponding flexible, event-study difference-in-differences version of the spillover result, following Equation (2). The figure also parallels the direct effect analysis reported in Figure 2. We see no evidence of a differential trend toward adopting a splitter treatment style between our treatment and control groups during the three years we can observe prior to the Medicare rule change $(t-6$ through $t-1)$. The pre-policy coefficients are close to zero and nowhere near statistically different from zero at conventional levels. The absence of a differential pre-trend prior to the Medicare policy change is consistent with the identifying assumption that the probability of allocating patients to both ASCs and HOPDs for a specific procedure would have continued to evolve in parallel across our treatment and control groups had it not been for the Medicare regulatory intervention in 2008. The differential behavior change for the policy-exposed surgeons begins only in the first postimplementation year, which then stabilizes at an elevated level by $t+4$ (i.e., the start of 2010).

More specifically, the event-time estimates in Table 3 and Figure 3 reveal that lifting the Medicare ASC prohibition for LC surgeries induced an approximately 3-percentage point increase in the probability that LC surgeons used ASCs and HOPDs for their commercial LC patients by the end of the first deregulation year $(t+1)$, which more than doubled over the next two postpolicy years to an 8-9 percentage point positive spillover effect. Compared to the pre-policy prevalence $(12.4 \%)$ of a splitter treatment style for policy-exposed (treatment group) physicians, the peak effect translates to an approximately $70 \%$ relative increase attributable to the Medicare rule change.

In Appendix Figure B3, we decompose the event study results from Figures 2 and 3 by separately estimating a version of Equation (2) for the treatment and control groups in isolation for each payer. Doing so reveals the time series for the outcomes for each of the respective DD 
groups. Figure B3 shows that the differential changes captured in Figures 2 and 3 are overwhelmingly driven by level changes among our policy exposed surgeons (i.e., the treatment group). In particular, with respect to the direct effect, Figure B3 shows event study estimates that are close to zero for the Medicare control group over our full analytic window. Therefore, the control group, while important in principle, is not in this case differencing-out strong underlying trends. With respect to the spillover effect, the commercial payer control group likewise demonstrates limited trending from 2005-2009, with slight declines in splitter prevalence during 2010 and 2011. Taken together, the time patterns of Figures 2 and 3 mirror each other, consistent with a direct effect of the Medicare policy spilling over onto the commercial market. We also note from Appendix Table B1 and Appendix Figure B4 that imposing a balanced panel of LC surgeons for the Medicare market produces the same inferences as those drawn from Table 2 and Figure 2. The only difference is a slightly larger effect magnitude, which is not surprising since this subset of the treatment group is the most consistently exposed to the Medicare market over time by definition.

\subsection{Robustness}

Our DD estimation applies relatively few restrictions to the analytic sample, especially with respect to which observations are included in the physician-procedure pairs that make up the control group. However, if an underlying trend in a particular control group procedure were driving the difference-in-differences result, this could be a cause for concern with the strategy. The flat pre- and post-trends in the pooled control group procedures in Appendix Figure B3 indicate that this is unlikely. Nonetheless, for completeness, we evaluate the possibility more systematically here. 
To do so, we first repeat our DD estimation for the direct (Medicare) and spillover (commercial) effects by systematically leaving out one of the non-LC procedures from the control group at a time and then re-estimating Equation (1). This process leads to 358 unique DD estimates for our coefficient of interest for the direct Medicare effects and 540 unique DD estimates for our coefficient of interest for the indirect, spillover effects onto the commercial payer market. We also perform a variant on this re-estimation approach with a randomly drawn set of control observations for each of our payer groups. Specifically, we implement bootstrap draws of a $50 \%$ sample (without replacement) of the available non-LC procedures belonging to the relevant payer group and then estimate Equation (1) using this subsample of non-LC control procedures. We repeat this sampling process for 1,000 iterations each for the direct and spillover results.

Figures 4 and 5 display the resulting DD estimate distributions for each exercise stratified by payer type. For Medicare (Figure 4), systematically excluding a control group procedure leaves the DD coefficients largely unchanged—even when the analytic sample sizes change by as much as 10,000 observations in some re-estimations (see Appendix Figure B5). The mass of the distribution in panel (a) of Figure 4 is almost entirely contained within [0.086, 0.090], a tight range around our 8.8-percentage point estimate (column 1 in Table 2). Likewise, using a randomly drawn subset of control group procedures (panel (b), Figure 4) creates a unimodal distribution of DD estimates around the observed effect from column 1 in Table 2 . The analogous robustness exercises for the commercially insured offer an identical pattern in Figure 5. Whether excluding non-LC procedures one-by-one (panel (a)) or bootstrapping random samples of control procedures (panel (b)), the DD estimates are tightly centered on the estimate from column 1 in Table 3 (i.e., a 6.5percentage point effect). 
The results displayed in Figures 4 and 5 therefore do not suggest that the presence or absence of any particular non-LC procedure in the control group has any meaningful impact on our main findings.

\subsection{Heterogeneity}

Do all physicians respond similarly when Medicare lifts its ban? Even conditioning estimation on effects within physicians over time by controlling for physician fixed effects, one might expect heterogeneous effects among the LC surgeons who have differential access to-or financial interests in-ASCs. We investigate this possibility next in a triple differences (DDD) approach that estimates heterogeneous coefficients on the basis of physician characteristics.

Our triple difference estimating equation is a simple extension of Equation (1) that introduces additional interaction terms:

$$
\begin{aligned}
\text { Splitter }_{q t}= & \delta_{1}\left(1\left(\text { Post }_{t}\right) \times 1\left(\text { LCSurgeon }_{q}\right)\right)+\delta_{2}\left(1\left(\text { Post }_{t}\right) \times 1\left(\text { LCSurgeon }_{q}\right) \times 1\left(\text { PhysCharac }_{q}\right)\right)+ \\
& \zeta\left(1\left(\text { Post }_{t}\right) \times 1\left(\text { PhysCharac }_{q}\right)\right)+\theta_{q}+\eta_{t}+\varepsilon_{q t}
\end{aligned}
$$

Here, Phys Charac is a time-invariant indicator that captures the heterogeneity of interest when interacted with the main difference-in-differences term (Post $\times$ LCSurgeon $)$. We estimate several versions of Equation (3), each with a different stratifying PhysCharac variable. The first version is an indicator for having any ASC use in 2007 for any non-LC procedures, irrespective of payer type. Pre-existing relationships with one or more ASCs could lower any adjustment frictions associated with shifting Medicare and non-Medicare LCs into ASC settings. The second version is an indicator of the individual physician holding an equity investment in one or more ASCs as of 
2007. ${ }^{31}$ Physicians with ownership stakes in ASCs-who would therefore receive some of the ASC facility fee through firm-level profit-sharing-could plausibly have stronger incentives to respond to any regulatory change affecting ASCs. The third version is an indicator equal to one if the physician completed medical school prior to $1990 .{ }^{32}$ Such LC surgeons would typically have been in independent (i.e., post-training) practice for at least 10 years prior to the start of our analytic window (i.e., 2005) and therefore may view changes to their practice patterns as more costly and/or less beneficial than physicians earlier in their career. ${ }^{33}$

The bottom rows of Table 4 list the means in our sample for the three stratifying PhysCharac variables, separately in the treatment and control groups. These reveal that the majority of our LC surgeons have some pre-existing ASC exposure by 2007 and have been in practice more than 10 years by the start of our study period. Just over $10 \%$ of them hold equity in one or more ASCs by 2007.

Results are reported in Table 4 for both the Medicare (direct effect) and the commercial (spillover) markets. Column 1 shows that existing use of an ASC leads to a stronger response within the Medicare market. The corresponding estimate in column 4 for the commercial market is directionally the same, though it lacks precision. The policy response appears largest for LC

\footnotetext{
${ }^{31}$ This information was generously shared by Beth Munnich and is part of her and her colleagues' ongoing research tied to physician ownership of ASCs- e.g., see Munnich et al. (2021).

32 These data are from the publicly available Physician Compare database provided by CMS. See here: https://data.cms.gov/provider-data/dataset/mj5m-pzi6. ASC ownership information and year of medical school graduation are at the National Provider Identification (NPI) level. The Florida discharge data only include this information starting in 2010; thus, we crosswalk the NPIs listed for our analytic sample of physicians in 2010 to their corresponding license numbers (i.e., our primary physician identifiers that are present in all years of data) to construct the variable.

${ }^{33}$ Not all physician licenses could be successfully matched to a unique and valid NPI. Additionally, some NPIs could not be successfully matched to the Physician Compare data. The first measure (2007 ASC use) relies solely on our primary data (i.e., the outpatient discharge records). The subsequent two measures (ASC ownership and medical school completion year) rely on external data sources that require linking to the discharge data. This processes necessarily leads to some analytic sample loss due to incomplete matches.
} 
surgeons that have ASC ownership investments. Columns 2 and 5, which stratify according to the physician's ASC ownership status, show that physicians who have an ownership stake in an ASC are more than three times as likely to begin performing some of their LCs at ASCs, compared to physicians without an ownership stake. That is true for both their Medicare and commercial patients. These findings indicate that physicians with strong financial ties to ASCs are the most sensitive to changes in Medicare policy when making treatment decisions for their Medicare and non-Medicare patients alike.

When stratifying the treatment group by medical school graduation date, the DDD estimates are negative and large for later career physicians; although, the t-statistics are only 1.87 and 1.73, respectively (columns 3 and 6). The pattern is at least suggestive of lower adjustment costs and/or greater perceived benefits of changing practice styles among physicians relatively newer to the profession. ${ }^{34}$

\section{Quality of Care and Secondary Spillovers}

\subsection{Patient postoperative discharge status}

A natural question, given the clear impacts on treatment setting choices established above, is whether shifting cases into ASC settings for the first time affected the quality of care. As noted in Section 3, our encounter data do not contain patient identifiers, which could otherwise allow for a detailed tracking of subsequent patient utilization (e.g., emergency department visits or other increases in post-operative care). However, each encounter record does have information on the patient's discharge status. To investigate impacts on patient outcomes, we define an outcome variable equal to one if the patient experiences a routine discharge to home with no further care

\footnotetext{
${ }^{34}$ Appendix Table B2 re-estimates all of Table 4 when imposing a consistent analytic sample across all estimations. The findings and interpretations align with those of Table 4.
} 
needs and zero otherwise (which could indicate, for example, that the patient required a transfer to another facility). We use Equation (1) and the same analytic samples from Section 4 to investigate this outcome.

The DD findings are presented in Table 5. It is important to note that more than $98 \%$ of all LC cases in either payer market conclude with a routine discharge to home. This is not surprising, given the "same day" business model belonging to the broader outpatient procedure market. The table shows no evidence of a change in the likelihood of LC surgeons' patients being routinely discharged following the procedure when these physicians begin routing patients to both treatment settings to perform the surgery. Both DD estimates (Medicare and commercial) are very small in magnitude and statistically insignificant. While we cannot conclude that there are no changes in care quality, among the one dimension that we can observe, we do not detect any effect.

\subsection{ASC use for untargeted procedures}

Tables 2 and 3, along with Figures 2 and 3, provide strong evidence that Medicare's 2008 policy change targeting LC surgeries affected the behavior of physicians performing the same surgery for non-Medicare (i.e., commercially insured) patients. However, Section 2 raised the possibility that the complementarities for physicians could extend beyond the focal (i.e., policy-targeted) procedure. For example, the convenience of scheduling a slate of procedures to be performed at a given ASC (e.g., over the course of an afternoon or entire day), rather than traveling between multiple facilities could influence physicians' willingness to newly allocate some of their untargeted procedures (e.g., tissues biopsies, colonoscopies, endoscopies, hernia repairs, etc.) to ASC treatment settings. Here we empirically test the possibility of "secondary spillovers" from removing the narrow Medicare ban on ASC-delivery for LC surgeries. 
To do so, we turn our attention to all non-targeted outpatient procedures-i.e., everything other than LC surgeries. We maintain the same treatment-control designations of physicians as before and use Equation (1). The key difference here is that rather than examining the LC surgeries of LC physicians and excluding their non-LC procedures, we examine their non-LC procedures and exclude their LC procedures. The sample restriction in terms of physicians is exactly the same as above (as in Table 3), and everything remains at the physician-procedure-payer-time level. ${ }^{35}$

We examine two outcomes. The first is the decision to split cases between settings, as above. The second outcome is motivated by the recognition that not all ASCs have the technical capacity to perform laparoscopic surgeries. It is therefore possible that the Medicare policy affected the type of ASC used (in terms of available surgical technology) by encouraging the policy-exposed physicians to migrate their non-LC procedures to facilities where LCs can be performed — even if they had already adopted a splitter treatment style for their non-LC procedures prior to 2008 . We investigate this possibility by constructing an additional binary outcome set equal to one only if the physician performs the relevant non-LC procedure within an ASC that has laparoscopic surgery technology. ${ }^{36}$ This second outcome could show secondary spillover effects even if the other (i.e., splitter) outcome does not.

Table 6 displays the estimates for secondary spillover effects, by payer type. Looking across all four columns in Table 6, we find no clear evidence that Medicare's targeted policy alters the treatment setting choices for affected physicians' untargeted procedures. The DD estimates are

\footnotetext{
${ }^{35}$ For the treatment group, the key difference is that each individual physician may have multiple panels (i.e., one for every specific, non-LC principal procedure where they have positive volume in all half-years), just like the control group physicians.

${ }^{36} \mathrm{We}$ are able to identify ASCs with this technology by leveraging our all-payer universe of outpatient procedures and a list of CPT codes for laparoscopic surgeries (all types) to determine if a given ASC in a given year has the technology. Of note, less than $30 \%$ of Florida ASCs perform any laparoscopic surgery cases (of any type for any payer) during the 2005-2011 period.
} 
uniformly small-to-modest in magnitude and statistically insignificant-though directionally positive for three of the four estimates (columns 2-4). Appendix Table B3 provides analogous results when aggregating all of a physician's non-LC procedures together, rather than creating the physician-by-procedure panels. Within Appendix Figures B6-B7, we show some descriptive trends in these outcomes when stratifying LC surgeons according to whether they started performing their LCs at ASCs following the 2008 Medicare rule change. There are suggestive indications of secondary spillovers, but we are unable to precisely detect any effects in our research design and with our sample size. Future work exploring Medicare rulemaking in other clinical contexts may be better suited for quantifying more dispersed impacts on untargeted procedures.

\section{Discussion}

It is well-established that the Medicare public insurance program is an influential force within the US healthcare system, affecting private medical markets in a variety of ways. Empirical attention to Medicare spillover effects has largely been devoted to provider responses to changes in Medicare financial incentives and to commercial plan price schedules for provider services. We examine a related but distinct question: whether Medicare rulemaking (in this case, a prohibition) impacts physicians' decision making and care provision for non-Medicare patients.

We focus on the physician choice to use an ASC for some patients, which builds on a prior literature aimed at understanding how and why physicians split caseloads across treatment settings (e.g., David and Neuman, 2011). We show that surgeons performing laparoscopic cholecystectomy procedures are more likely to divide their relevant Medicare patients between ASCs and HOPDs once the ASC-delivery ban is lifted in 2008, with a growing effect over the subsequent three years. Our contribution is to demonstrate that the Medicare rule change crowds 
in (rather than crowds out) commercial laparoscopic cholecystectomy surgeries performed in ASCs, with affected physicians becoming up to $70 \%$ more likely to split their commercial patients needing the same procedure across hospital and ASC settings, shortly after the procedure-specific Medicare policy is implemented. Physicians with existing ties (especially financial) to ASCs also demonstrate the strongest response, and we find no evidence suggesting more frequent surgical complications as ASCs begin substituting for HOPD delivery.

The direct effect of the policy includes not only the expansion of the facility choice set for patients, but also cost savings accruing to the Medicare program and Medicare patients (via cost sharing), since ASC facility fees are generally set below $60 \%$ of the corresponding HOPD facility fees for the same procedure code. ${ }^{37}$ In terms of the share of Medicare procedures affected, $8 \%$ of Medicare LC procedures performed by the physicians in our analytic sample take place in ASCs by the final year of our study period (2011) - all of which can be attributed to the removal of the ban. An analogously straightforward calculation is not available for commercial laparoscopic cholecystectomy cases because some increases in ASC use for LCs for the commercially insured between the pre- and post-periods could have occurred absent any Medicare policy change. Descriptively, we observe a commercially insured LC ASC use increase from $5.2 \%$ to $8.2 \%$ among the treatment group between the pre- and post-periods (Appendix Table B4). ${ }^{38}$

\footnotetext{
${ }^{37}$ Because commercial insurer transaction prices are typically much higher than Traditional Medicare fee schedules - e.g., about double for the common procedures analyzed by Cooper et al. (2019) - the per-procedure cost savings in commercial sector could be even larger.

${ }^{38}$ To further examine the intensive margin while trying to appropriately control for any underlying trends, we return to the same difference-in-differences regression from Equation (2). We replace the payer-specific splitter dependent variable in Equation (2) with the inverse hyperbolic sine-transformed fraction of cases of each procedure sent to ASCs. The unit of observation remains the physician-procedure-period level, and the control group remains all procedures by physicians who do not perform LCs, just as in Figures 2 and 3. The inverse hyperbolic sine transformation is meant to better match the control and treatment group trends because the control groups (non-LC procedures) have very different underlying baseline rates of ASC use from each other and from the treatment group, which can challenge a comparison of level changes. Appendix Figure B8 reports these supplementary event study results for both the direct effect on Medicare volume and the spillover effect on commercial volume. Panel (a) plots the difference-in-differences coefficients for the volume of ASC use in Medicare, showing a large relative change in
} 
In sum, restrictions imposed by the public insurer can affect treatment setting decisions for Medicare and non-Medicare patients alike, which may be at odds with non-Medicare patients' and payers' underlying preferences. Our findings also imply that Medicare's restrictions may have held back opportunities for other parties to cut healthcare costs outside of the Medicare program.

The physician behavior changes we establish in Section 4 are also consistent with crosspayer complementarities, as described in Section 2. However, as previously noted, the findings could plausibly align with private insurer rule-following as well. ${ }^{39}$ In principle, a test of whether policy-exposed physicians who never treat Medicare patients with the targeted procedure still change behavior for their commercial patients could help differentiate between physician-driven complementarities versus insurer-driven rule following explanations. Complementarities between Medicare and non-Medicare treatment decisions cannot exist among surgeons that do not participate in the Medicare market. In practice, only a single surgeon from our treatment group physicians has zero Medicare business for the affected procedure, rendering such a falsification

ASC use for LC surgeons for Medicare patients, but which is driven by a small absolute change (5\%). Panel (b) plots the analogous coefficients for the commercially insured patients, which indicate that overall ASC use for LCs among commercially insured patients increases by a little over $10 \%$ on average, relative to the six months preceding the Medicare policy change and relative to the physician-procedure pairs comprising our control group. For both the Medicare and commercial regressions, the early part of our analytic window demonstrates poor alignment between the treatment and control units, driven by underlying trends in the controls. Overall, these results are less precise than our main splitter specification and less well-behaved, with an imperfect match between the control and treatment trends. We therefore treat these results as only suggestive. For completeness, we also report a specification that uses a non-transformed version of the dependent variable, \%ASC use. Appendix Figure B9 shows that there is a strong differential trend in this outcome, driven by a non-flat time trend in the control that is present throughout the analytic window.

${ }^{39}$ For example, related evidence exists for commercial and public insurer interdependence tied to evaluations and coverage determinations for advancements in care delivery and medical technology (e.g., see Garber 2001; Chambers et al. 2015). Examples also exist of patient advocacy efforts centered around securing Medicare coverage for a specific treatment in order to spur commercial coverage of the same treatment for the nonelderly population (e.g., see Kaiser Health News 2019), and previous research even posited that commercial payers might eschew ASC-delivery for laparoscopic cholecystectomies, specifically, due to Medicare's pre-2008 regulatory stance and the implied safety concerns (see Paquette et al. 2008). 
exercise infeasible. ${ }^{40}$ In Appendix $\mathrm{C}$, we take a different approach toward understanding the importance of rule following. There, we leverage a supplementary claims-level database (Marketscan) of more than 640,000 LC surgeries paid for by employer-sponsored health insurers across the US from 2005 to 2012. These data, in which individual carriers and plans are separately identifiable, offer no clear indication that carriers or plans newly permit ASC delivery for this specific surgery only after Medicare's 2008 rule change. Thus, we fail to find clear empirical evidence supporting a rule-following interpretation, though we cannot rule it out.

Irrespective of the underlying mechanism or mechanisms in play, we offer novel evidence of substantive spillover effects on physician behavior from regulatory decisions made by the Medicare program. Our results are of interest not only as a case study of the specific regulatory change involved, but also as evidence of the likely impacts on commercial care provision for the thousands of other non-price Medicare regulations that govern how or where procedures may be performed in Medicare - with the magnitudes of such spillovers likely to depend on the complexity of the service being deregulated and accompanying adjustment frictions. ${ }^{41}$ Our findings therefore shed light on an important, but underexplored determinant of physician practice patterns and treatment styles: nonprice public insurer regulations. Such externalities deserve greater attention as the Medicare program continues to adjust regulatory parameters tied to outpatient surgery (e.g., changes to the "inpatient only" (IPO) list and related ASC permissions) to offer more convenient and lower cost treatment options for beneficiaries. ${ }^{42}$ While Medicare rulemaking does not

\footnotetext{
${ }^{40}$ In other words, 386 out of 387 of the surgeons in our analytic sample pertaining to the spillover (commercial payer) analyses have non-zero Medicare LC surgeries at some point during our study period.

${ }^{41}$ For instance, we examined a moderate-complexity procedure, with specific capital requirements (i.e., laparoscopic surgery technology). Only a minority of existing ASCs had the requisite capital investments prior to Medicare removing its ASC ban for laparoscopic cholecystectomies and few new ASCs were created after 2008 (e.g., see Munnich and Richards 2021).

${ }^{42}$ See Meyer (2017) and Brady (2020), for example. Related and additional information from the Centers for Medicare \& Medicaid Services (CMS) on the CY 2021 Medicare Hospital Outpatient Prospective Payment System
} 
explicitly account for externalities on non-Medicare patients, our findings indicate that effects on other payer groups could be significant and influence the overall costs and benefits of such contemporary policy decisions.

and Ambulatory Surgical Center Payment System Final Rule (CMS-1736-FC) can be found here: https://www.cms.gov/newsroom/fact-sheets/cy-2021-medicare-hospital-outpatient-prospective-payment-systemand-ambulatory-surgical-center-0. See here for an example of some recent Medicare policy shifts affecting ASC treatment permissions: $\underline{\mathrm{https}}$ ://www.modernhealthcare.com/outpatient/medicare-payment-change-will-shiftlucrative-heart-procedures-out-hospital. 


\section{References}

Aouad, Marion, Timothy T. Brown, and Christopher M. Whaley. 2019. "Reference Pricing: The Case of Screening Colonoscopies." Journal of Health Economics, 65, 246-259.

Baicker, Katherine, Michael E. Chernew, and Jacob A. Robbins. 2013. "The Spillover Effects of Medicare Managed Care: Medicare Advantage and Hospital Utilization.” Journal of Health Economics, 32 (6): 1289-1300.

Baicker, Katherine, and Jacob A Robbins. 2015. "Medicare Payments and System-Level Health Care Use: The Spillover Effects of Medicare Managed Care." American Journal of Health Economics, 1 (4): 399-431.

Baker, Laurence C., M. Kate Bundorf, and Daniel P. Kessler. 2019. "Competition in Outpatient Procedure Markets." Medical Care, 57 (1): 36-41.

Barnett, Michael L., Andrew Olenski, and Adam Sacarny. 2020. "Common Practice: Spillovers from Medicare on Private Health Care.” NBER Working Paper Series, w27270, http://www.nber.org/papers/w27270.

Bian, John, and Michael A. Morrisey. 2007. "Free-Standing Ambulatory Surgery Centers and Hospital Surgery Volume.” Inquiry, 44: 200-210.

Bond, Amelia M., and Chapin White. 2013. "Massachusetts Coverage Expansion Associated with Reduction in Primary Care Utilization among Medicare Beneficiaries." Health Services Research, 48 (6): 1826-1839.

Brady, Michael. 2020. "CMS' Plan to End Inpatient-Only List Worries Providers." Modern Healthcare, October, 6, 2020, Crain Communications Inc. Available here: https://www.modernhealthcare.com/payment/cms-plan-end-inpatient-only-list-worriesproviders.

Callison, Kevin. 2016. "Medicare Managed Care Spillovers and Treatment Intensity." Health Economics, 25: 873-887.

Carey, Colleen M., Sarah Miller, and Laura R. Wherry. 2020. "The Impact of Insurance Expansions on the Already Insured: The Affordable Care Act and Medicare." NBER Working Paper Series, w25153, http://www.nber.org/papers/w25153.

Carey, Kathleen. 2015. "Price Increases Were Much Lower in Ambulatory Surgery Centers than Hospital Outpatient Departments in 2007-12." Health Affairs, 34 (10): 1738-1744. 
Carey, Kathleen. 2017. "Ambulatory Surgery Centers and Prices in Hospital Outpatient Departments." Medical Care Research and Review, 74 (2): 236-248.

Carey, Kathleen, James F. Burgess Jr., and Gary J. Young. 2011. "Hospital Competition and Financial Performance: The Effects of Ambulatory Surgery Centers." Health Economics, 20: 571-581.

Chambers, James D., Matthew Chenoweth, Teja Thorat, and Peter J. Neumann. 2015. "Private Payers Disagree with Medicare over Medical Device Coverage about Half the Time." Health Affairs, 34 (8): 1376-1382.

Chen, Alice J., Michael R. Richards, Christopher M. Whaley, and Xiaoxi Zhao. 2022. "The Extent of Externalities from Medicare Payment Policy.” American Journal of Health Economics, online ahead of print.

Chernew, Michael, Philip DeCicca, and Robert Town. 2008. "Managed Care and Medical Expenditures of Medicare Beneficiaries." Journal of Health Economics, 27 (6): 14511461.

Clemens, Jeffrey, and Joshua D. Gottlieb. 2014. "Do Physicians' Financial Incentives Affect Medical Treatment and Patient Health?" American Economic Review, 104 (4): 13201349.

Clemens, Jeffrey, and Joshua D. Gottlieb. 2017. "In the Shadow of a Giant: Medicare's Influence on Private Physician Payments." Journal of Political Economy, 125 (1): 1-39.

Clemens, Jeffrey, Joshua D. Gottlieb, and Tímea Laura Molnár. 2017. "Do Health Insurers Innovate? Evidence from the Anatomy of Physician Payments." Journal of Health Economics, 55: 153-167.

Cooper, Zack, Stuart V. Craig, Martin Gaynor, and John Van Reenen. 2019. "The Price Ain't Right? Hospital Prices and Health Spending on the Privately Insured." Quarterly Journal of Economics, advanced access September 2018: 51-107.

Courtemanche, Charles and Michael Plotzke. 2010. "Does Competition from Ambulatory Surgical Centers Affect Hospital Surgical Output?" Journal of Health Economics, 29: 765-773. 
Currie, Janet, W. Bentley MacLeod, and Jessica Van Parys. 2016. "Provider Practice Style and Patient Health Outcomes: The Case of Heart Attacks." Journal of Health Economics, 47: 64-80.

Cutler, David, Jonathan Skinner, Ariel Dora Stern, and David Wennberg. 2019. "Physician Beliefs and Patient Preferences: A New Look at Regional Variation in Health Care Spending." American Economic Journal: Economic Policy, 11 (1): 192-221.

David, Guy and Mark D. Neuman. 2011. "Physician Division of Labor and Patient Selection for Outpatient Procedures." Journal of Health Economics, 30(2): 381-391.

Dyrda, Laura. 2017. "39\% of ASCs are 15+ years old, $92 \%$ have physician ownership: 14 statistics on ASCs." Becker's ASC Review, October 9. Available at https://www.beckersasc.com/benchmarking/39-of-ascs-are-15-years-old-92-havephysician-ownership-14-statistics-on-ascs.html.

Einav, Liran, Amy Finkelstein, Yunan Ji, and Neale Mahoney. 2020. "Randomized Trial Shows Healthcare Payment Reform Has Equal-Sized Spillover Effects on Patients Not Targeted by Reform." Proceedings of the National Academy of Sciences, www.pnas.org/cgi/doi/10.1073/pnas.2004759117: 1-9. Last Accessed: August 2020.

Epstein, Andrew J. and Sean Nicholson. 2009. "The Formation and Evolution of Physician Treatment Styles: An Application to Cesarean Sections." Journal of Health Economics, 28 (6): 1126-1140.

Frank, Richard G. and Richard Z. Zeckhauser. 2007. "Custom-Made versus Ready-to-Wear Treatments: Behavioral Propensities in Physicians' Choices." Journal of Health Economics, 26: 1101-1127.

Garber, Alan M. 2001. "Evidence-Based Coverage Policy.” Health Affairs, 20 (5): 62-82.

Garthwaite, Craig L. 2012. "The Doctor Might See You Now: The Supply Side Effects of Public Health Insurance Expansions.” American Economic Journal: Economic Policy, 4 (3): 190-215.

Glied, Sherry and Joshua Graff Zivin. 2002. "How Do Doctors Behave When Some (But Not All) of Their Patients Are in Managed Care?" Journal of Health Economics, 21: 337-353. 
Glied, Sherry, and Kai Hong. 2018. "Health Care in a Multi-Payer System: Spillovers of Health Care Service Demand among Adults under 65 on Utilization and Outcomes in Medicare." Journal of Health Economics, 60: 165-176.

Grisel, Jedidiah and Ellis Arjmand. 2009. "Comparing Quality at an Ambulatory Surgery Center and a Hospital-Based Facility." Otolaryngology-Head and Neck Surgery, 141 (6): 701709.

Grytten, Jostein, and Rune Sørensen. 2003. "Practice Variation and Physician-Specific Effects." Journal of Health Economics, 22 (3): 403-418.

Hall, Margaret J., Alexander Schwartzman, Jin Zhang, Xiang Liu, and Division of Health Care Statistics. 2017. "Ambulatory Surgery Data from Hospitals and Ambulatory Surgery Centers: United States, 2010.” National Health Statistics Reports, No. 102, 28 February 2017, Centers for Disease Control and Prevention; US Department of Health and Human Services.

He, Daifeng, and Jennifer M. Mellor. 2012. "Hospital Volume Responses to Medicare's Outpatient Prospective Payment System: Evidence from Florida." Journal of Health Economics, 31 (5): 730-743.

He, Daifeng, and Jennifer M. Mellor. 2013. "Do Changes in Hospital Outpatient Payments Affect the Setting of Care?" Health Services Research, 48 (5): 1593-1616.

He, Daifeng, Melissa McInerney, and Jennifer M. Mellor. 2015. "Physician Responses to Rising Local Unemployment Rates: Healthcare Provision to Medicare and Privately Insured Patients." Journal of Health Economics, 40: 97-108.

Joynt, Karen E., David Chan, E. John Orav, and Ashish K. Jha. 2013. "Insurance Expansion in Massachusetts Did Not Reduce Access among Previously Insured Medicare Patients." Health Affairs, 32 (3): 571-578.

Joynt, Karen E., David C. Chan, Jie Zheng, E. John Orav, and Ashish Jha. 2015. "The Impact of Massachusetts Health Care Reform on Access, Quality, and Costs of Care for the Already-Insured." Health Services Research, 50 (2): 599-613.

Kaiser Health News (KHN), reporting by Carmen Heredia Rodriguez. Compression Garments Can Ease Lymphedema. Covering Costs? Not So Easy. October 23, 2019. Available here: https://khn.org/news/compression-garments-lymphadema-insurance-coverage-costs/ 
Lynk, William J. and Carina S. Longley. 2002. "The Effect of Physician-Owned Surgicenters on Hospital Outpatient Surgery." Health Affairs, 21 (4): 215-221.

MacKinnon, James G. and Matthew D. Webb. 2017. "Wild Bootstrap Inference for Wildly Different Cluster Sizes." Journal of Applied Econometrics, 32: 233-254.

McGuire, Thomas G., and Mark V. Pauly. 1991. "Physician Response to Fee Changes with Multiple Payers." Journal of Health Economics, 10 (4): 385-410.

McInerney, Melissa, Jennifer M. Mellor, and Lindsay M. Sabik. 2017. "The Effects of State Medicaid Expansions for Working-Age Adults on Senior Medicare Beneficiaries." American Economic Journal: Economic Policy, 9 (3): 408-438.

MedPAC. 2019. Report to the Congress: Medicare Payment Policy. Chapter 5 (Ambulatory Surgical Center Services). March 2019. Available here:

http://www.medpac.gov/docs/defaultsource/reports/mar19_medpac_ch5_sec.pdf?sfvrsn $=0$.

Meredith, Catherine. 2008. “General Surgery's Promise.” Outpatient Surgery, February 2008, Herrin Publishing Partners LP.

Meyer, Harris. 2017. "Hospitals Leery of CMS Proposal to Pay for Joint Replacements in ASCs." Modern Healthcare, August, 5, 2017, Crain Communications Inc. Available here: https://www.modernhealthcare.com/article/20170805/NEWS/170809931/hospitals-leeryof-cms-proposal-to-pay-for-joint-replacements-in-ascs.

Meyers, David J., Cyrus M. Kosar, Momotazur Rahman, Vincent Mor, and Amal N. Trivedi. 2019. "Association of Mandatory Bundled Payments for Joint Replacements with Use of Postacute Care among Medicare Advantage Enrollees.” JAMA Network Open, 2 (12): 112.

Molitor, David. 2018. "The Evolution of Physician Practice Style: Evidence from Cardiologist Migration." American Economic Journal: Economic Policy, 10 (1): 326-356.

Munnich, Elizabeth L. and Stephen T. Parente. 2014. "Procedures Take Less Time at Ambulatory Surgery Centers, Keeping Costs Down and Ability to Meet Demand Up." Health Affairs, 33 (5): 764-769.

Munnich, Elizabeth L., and Stephen T. Parente. 2018. "Returns to Specialization: Evidence from the Outpatient Surgery Market." Journal of Health Economics, 57: 147-167. 
Munnich, Elizabeth L., Michael R. Richards, Christopher M. Whaley, and Xiaoxi Zhao. 2021. "Raising the Stakes: Physician Facility Investments and Provider Agency." SSRN Working Paper Series. Available at SSRN: https://ssrn.com/abstract=3845073.

Munnich, Elizabeth L., and Michael R. Richards. 2021. "Long-Run Growth of Ambulatory Surgery Centers 1990-2015 and Medicare Payment Policy." Forthcoming, Health Services Research.

Newhouse, Joseph P. and M. Susan Marquis. 1978. "The Norms Hypothesis and the Demand for Medical Care.” Journal of Human Resources, 13 Supplement: 159-182.

OR Manager. 2007. “CMS Sets Final 2008 ASC Payment Rates.” OR Manager Inc. 23 (12): 1-3. https://www.ormanager.com/wpcontent/uploads/2012/06/ORMVol23No12ASCcmsPaymentRates.pdf

Paquette, Ian M., Douglas Smink, and Samuel R.G. Finlayson. 2008. "Outpatient Cholecystectomy at Hospitals Versus Freestanding Ambulatory Surgical Centers." Journal of the American College of Surgeons, 206 (2): 301-305.

Phelps, Charels E. 2000. "Information Diffusion and Best Practice Adoption." In Handbook of Health Economics, Vol. 1A, edited by Anthony J. Culyer and Joseph P. Newhouse, 22364. Amsterdam: North-Holland.

Plotzke, Michael and Charles Courtemanche. 2011. "Does Procedure Profitability Impact Whether an Outpatient Surgery is Performed at an Ambulatory Surgery Center or Hospital?" Health Economics, 20 (7): 817-830.

Polychronidis, Alexandros, Prodromos Laftsidis, Anastasios Bounovas, and Constantinos Simopoulos. 2008. "Twenty Years of Laparoscopic Cholecystectomy: Philippe MouretMarch 17, 1987." Journal of the Society of Laparoendoscopic Surgeons, 12: 109-111.

Richards, Michael R., and Sebastian Tello-Trillo. 2019. "Public Spillovers from Private Insurance Contracting: Physician Responses to Managed Care." American Economic Journal: Economic Policy, 11 (4): 375-403.

Sloan, Frank A., Michael A. Morrisey, and Joseph Valvona. 1988. "Effects of the Medicare Prospective Payment System on Hospital Cost Containment: An Early Appraisal." Milbank Quarterly, 66 (2): 191-220. 
Sood, Neeraj and Chistopher M. Whaley. 2019. "Reverse Reference Pricing: Rewarding Patients for Reducing Medicare Costs." Health Affairs Blog, June 7, 2019. https://www.healthaffairs.org/do/10.1377/hblog20190604.509495/full/.

Trish, Erin, Paul Ginsburg, Laura Gascue, and Geoffrey Joyce. 2017. "Physician Reimbursement in Medicare Advantage Compared with Traditional Medicare and Commercial Health Insurance.” JAMA Internal Medicine, 177 (9): 1287-1295.

Weber, Ellerie. 2014. "Measuring Welfare from Ambulatory Surgery Centers: A Spatial Analysis of Demand for Healthcare Facilities." The Journal of Industrial Economics, 62(4): 591-631.

Whaley, Christopher M., and Timothy T. Brown. 2018. "Firm Responses to Targeted Consumer Incentives: Evidence from Reference Pricing for Surgical Services." Journal of Health Economics, 61: 111-133.

White, Chapin. 2013. "Contrary to Cost-Shift Theory, Lower Medicare Hospital Payment Rates for Inpatient Care Lead to Lower Private Payment Rates." Health Affairs, 32 (5): 935943.

White, Chapin. 2014. "Cutting Medicare Hospital Prices Leads to a Spillover Reduction in Hospital Discharges for the Nonelderly.” Health Services Research, 49 (5): 1578-1595.

Wilcock, Andrew D., Michael L. Barnett, J. Michael McWilliams, David C. Grabowski, and Ateev Mehrotra. 2020. “Association between Medicare's Mandatory Joint Replacement Bundled Payment Program and Post-Acute Care Use in Medicare Advantage." JAMA Surgery, 155 (1): 82-84.

Yee, Christine A. 2011. "Physicians on Board: An Examination of Physician Financial Interests in ASCs Using Longitudinal Data." Journal of Health Economics, 30: 904-918.

Yip, Winnie. 1998. "Physician Response to Medicare Fee Reductions: Changes in the Volume of Coronary Artery Bypass Graft (CABG) Surgeries in the Medicare and Private Sectors." Journal of Health Economics, 17 (6): 675-699.

Yurukoglu, Ali, Eli Liebman, and David B. Ridley. 2017. "The Role of Government Reimbursement in Drug Shortages." American Economic Journal: Economic Policy, 9 (2): 348-382. 


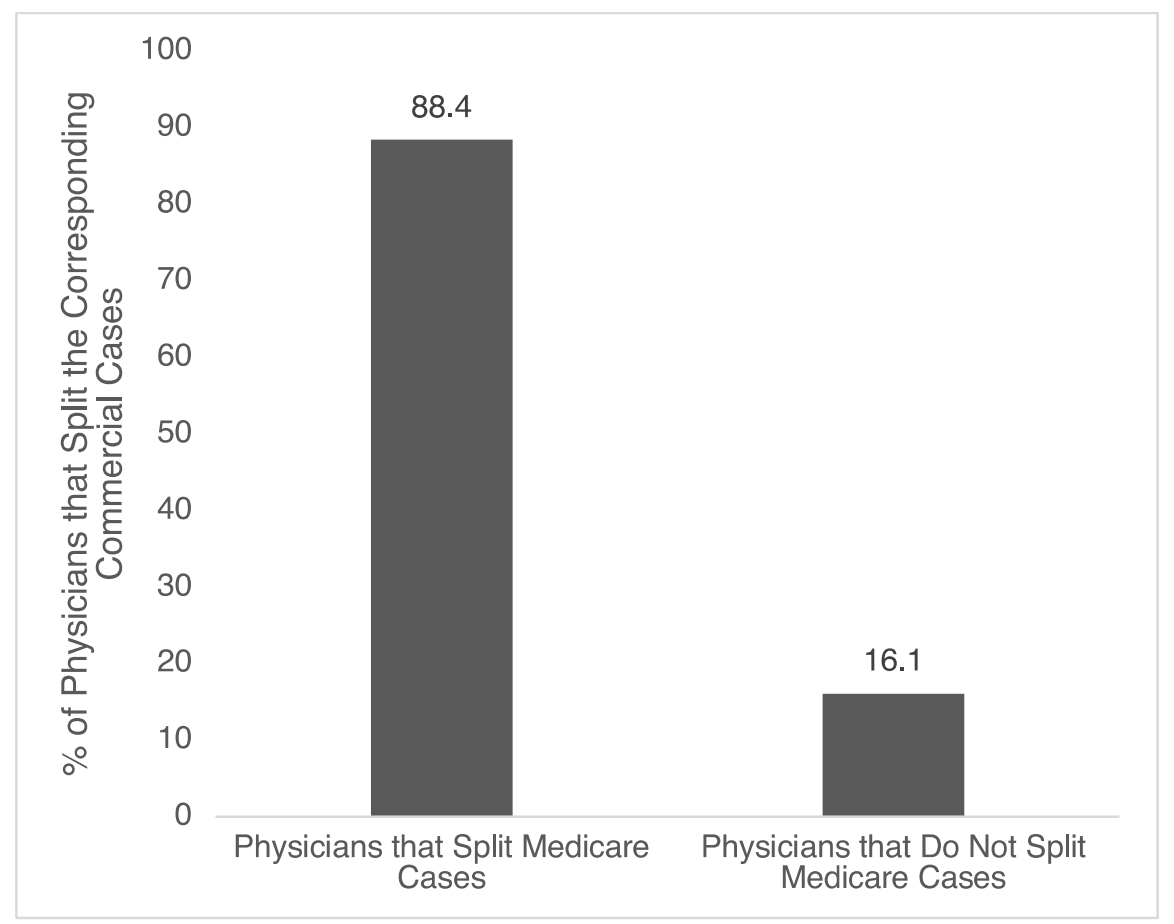

Fig 1. Cross-Sectional Correspondence in Splitting Cases Between ASCs And HOPDs Within-Physicians, Across Their Medicare and Commercial Patients

Notes: The data are from the universe of ambulatory procedure discharge records in Florida, and observations are at the physician-byprocedure level. Splitting is defined as having positive volume at both ASC and HOPD treatment settings for the physician-procedure pair. The data are restricted to 2007 and principal procedures recorded in the discharge records. 
Table 1: Summary Stats by Procedure and Payer Type

\begin{tabular}{|c|c|c|c|c|}
\hline & \multicolumn{2}{|c|}{ Laparoscopic Cholecystectomy } & \multicolumn{2}{|c|}{ All Other Procedures } \\
\hline & Commercial & Medicare & Commercial & Medicare \\
\hline & Mean (SD) & Mean (SD) & Mean (SD) & Mean (SD) \\
\hline \multirow{3}{*}{$\begin{array}{l}\text { Age } \\
\text { Number of } \\
\text { Comorbidities }\end{array}$} & $44.8(12.6)$ & $69.3(10.8)$ & $48.8(16.7)$ & $72.8(10.2)$ \\
\hline & $2.1(2.2)$ & $4.1(2.8)$ & $1.6(1.9)$ & $2.0(2.5)$ \\
\hline & $(\%)$ & $(\%)$ & $(\%)$ & $(\%)$ \\
\hline Female & 77.5 & 66.4 & 57.6 & 55.0 \\
\hline White & 77.3 & 85.8 & 76.3 & 85.8 \\
\hline $\mathrm{N}$ & 113,524 & 32,201 & $9,242,328$ & $7,200,586$ \\
\hline $\begin{array}{l}\% \text { Performed in } \\
\text { ASC, } 2005-2007\end{array}$ & 6.0 & -- & 50.1 & 53.2 \\
\hline \multicolumn{5}{|c|}{$\begin{array}{l}\text { Notes: Summary statistics include all ambulatory (outpatient) procedural discharge records } \\
\text { from Florida during the } 2005-2011 \text { period. The data are stratified by payer type (i.e., } \\
\text { Commercial versus Medicare) but not setting type (i.e., ASC versus HOPD). The } \\
\text { commercial payer group excludes Medicare Advantage, and the Medicare payer group is } \\
\text { restricted to traditional (fee-for-service) Medicare patients. Examples of highly common, } \\
\text { non-LC outpatient procedures include: cataract removals, colonoscopies, endoscopies, } \\
\text { hernia repairs, skin biopsies, and spinal injections. }\end{array}$} \\
\hline
\end{tabular}


Table 2: Diff-in-Diff Estimates for the Direct Effect of Medicare Regulation on the Likelihood of Using Both ASC and HOPD Treatment Settings for Medicare Patients

\begin{tabular}{|c|c|c|}
\hline & Pr(Splitter) & Pr(Splitter) \\
\hline & $(1)$ & (2) \\
\hline Post $X$ LC Surgeon & $\begin{array}{l}0.088^{* * *} \\
(0.013)\end{array}$ & \\
\hline$(t-6) \times$ LC Surgeon & & $\begin{array}{l}-0.023 * * \\
(0.010)\end{array}$ \\
\hline$(t-5) \times$ LC Surgeon & & $\begin{array}{c}0.008 \\
(0.012)\end{array}$ \\
\hline$(t-4) \times$ LC Surgeon & & $\begin{array}{l}-0.001 \\
(0.011)\end{array}$ \\
\hline$(t-3) \times$ LC Surgeon & & $\begin{array}{c}0.004 \\
(0.011)\end{array}$ \\
\hline$(t-2) \times$ LC Surgeon & & $\begin{array}{c}0.002 \\
(0.010)\end{array}$ \\
\hline$(t+0) \times$ LC Surgeon & & $\begin{array}{l}0.052 * * * \\
(0.016)\end{array}$ \\
\hline$(t+1) \times$ LC Surgeon & & $\begin{array}{l}0.062 * * * \\
(0.016)\end{array}$ \\
\hline$(t+2) \times$ LC Surgeon & & $\begin{array}{l}0.073 * * * \\
(0.018)\end{array}$ \\
\hline$(t+3) \times$ LC Surgeon & & $\begin{array}{l}0.075^{* * *} \\
(0.019)\end{array}$ \\
\hline$(t+4) \times$ LC Surgeon & & $\begin{array}{l}0.098 * * * \\
(0.018)\end{array}$ \\
\hline$(t+5) \times$ LC Surgeon & & $\begin{array}{l}0.122 * * * \\
(0.020)\end{array}$ \\
\hline$(t+6) \times$ LC Surgeon & & $\begin{array}{l}0.084 * * * \\
(0.018)\end{array}$ \\
\hline$(t+7) \times$ LC Surgeon & & $\begin{array}{l}0.130 * * * \\
(0.020)\end{array}$ \\
\hline Physician-Procedure FE & Yes & Yes \\
\hline Half-Year FE & Yes & Yes \\
\hline $\begin{array}{l}\text { Unique Physician- } \\
\text { Procedure Pairs }\end{array}$ & 7,733 & 7,733 \\
\hline Observations $(\mathrm{N})$ & 107,458 & 107,458 \\
\hline \multicolumn{3}{|c|}{$\begin{array}{l}\text { Notes: "Splitter" indicates that the physician provides at least some } \\
\text { of the relevant procedure cases at both ASC and HOPD settings to } \\
\text { their Medicare patients. "LC Surgeon" is our treatment group (i.e., } \\
\text { laparoscopic cholecystectomy surgeons). The control group is } \\
\text { composed of all provider-procedure combinations among non-LC } \\
\text { physicians observed over our analytic window. } \\
* * \text { P value at } 0.05 * * * \mathrm{P} \text { value at } 0.01 \text {, standard errors clustered at } \\
\text { the physician level }\end{array}$} \\
\hline
\end{tabular}




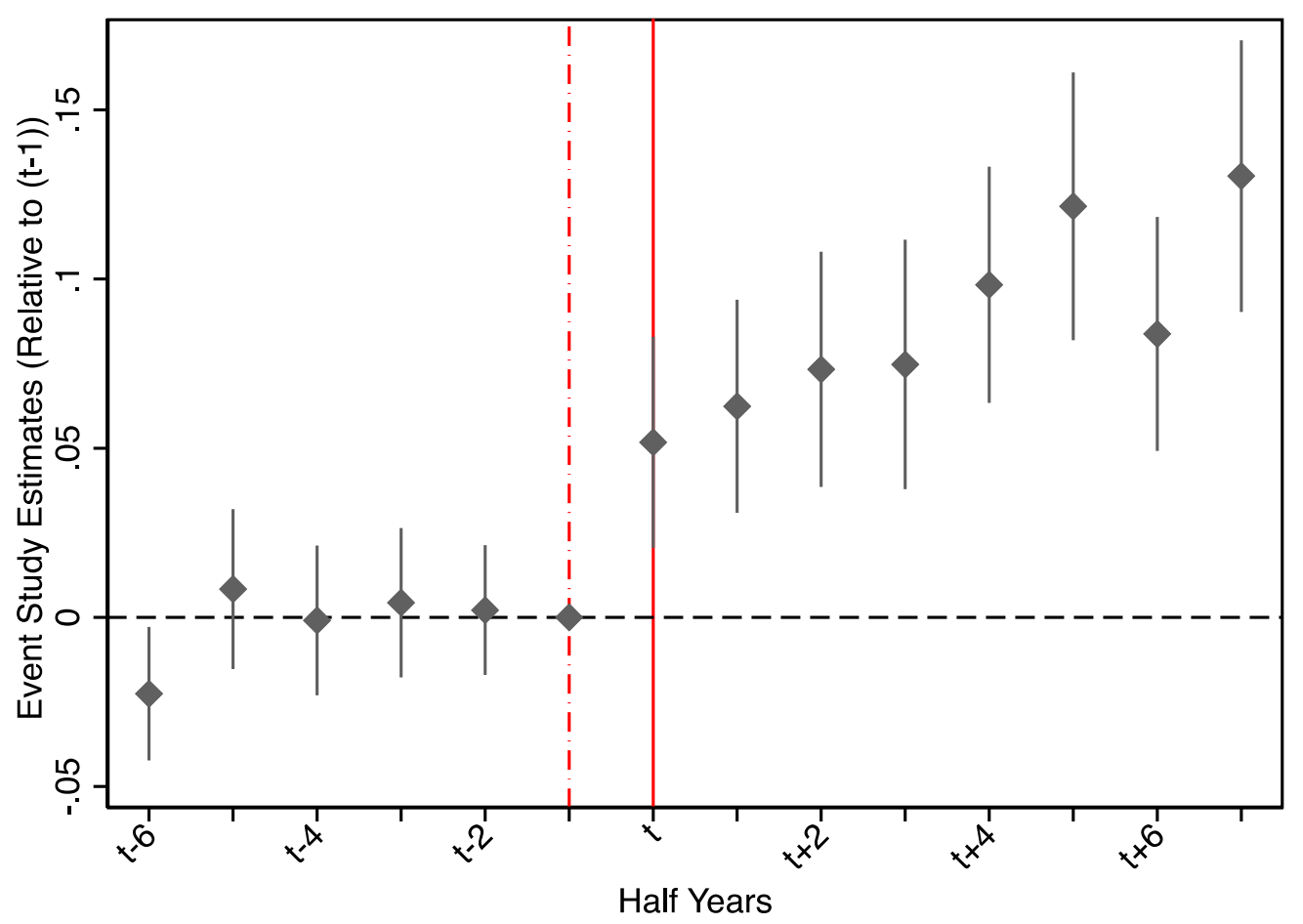

Fig 2. Direct Effect of Medicare Policy Change That Allowed LC Surgeries in ASC Settings

Notes: The treatment group is all LC surgeons consistently observed over our 2005-2011 period in the commercial market. "Splitter" indicates that the physician provides at least some of the relevant procedure cases at both ASC and HOPD settings. The control group is composed of all provider-procedure combinations among non-LC physicians observed over our analytic window. Time periods are in half-year increments. The variables are constructed based only on traditional Medicare (i.e., fee-for-service) cases for the physicians in the sample. $\mathrm{N}=107,458$, unique provider-procedure fixed effects $=7,733$. 
Table 3: Diff-in-Diff Estimates for the Spillover Effect of Medicare Regulation on the Likelihood of Using Both ASC And HOPD Treatment Settings for Commercial Patients

\begin{tabular}{|c|c|c|}
\hline & $\operatorname{Pr}($ Splitter) & $\operatorname{Pr}($ Splitter) \\
\hline & $(1)$ & (2) \\
\hline Post $\times$ LC Surgeon & $\begin{array}{l}0.065^{* * *} \\
(0.013)\end{array}$ & \\
\hline$(t-6) \times$ LC Surgeon & & $\begin{array}{l}-0.008 \\
(0.017)\end{array}$ \\
\hline$(t-5) \times$ LC Surgeon & & $\begin{array}{c}0.006 \\
(0.018)\end{array}$ \\
\hline$(t-4) \times$ LC Surgeon & & $\begin{array}{c}0.005 \\
(0.016)\end{array}$ \\
\hline$(t-3) \times$ LC Surgeon & & $\begin{array}{l}-0.014 \\
(0.016)\end{array}$ \\
\hline$(t-2) \times$ LC Surgeon & & $\begin{array}{c}0.005 \\
(0.014)\end{array}$ \\
\hline$(t+0) \times$ LC Surgeon & & $\begin{array}{c}0.015 \\
(0.013)\end{array}$ \\
\hline$(t+1) \times$ LC Surgeon & & $\begin{array}{l}0.036^{* *} \\
(0.016)\end{array}$ \\
\hline$(t+2) \times$ LC Surgeon & & $\begin{array}{l}0.051 * * * \\
(0.016)\end{array}$ \\
\hline$(t+3) \times$ LC Surgeon & & $\begin{array}{l}0.063 * * * \\
(0.018)\end{array}$ \\
\hline$(t+4) \times$ LC Surgeon & & $\begin{array}{l}0.083 * * * \\
(0.019)\end{array}$ \\
\hline$(t+5) \times$ LC Surgeon & & $\begin{array}{l}0.089 * * * \\
(0.020)\end{array}$ \\
\hline$(t+6) \times$ LC Surgeon & & $\begin{array}{l}0.088^{* * *} \\
(0.020)\end{array}$ \\
\hline$(t+7) \times$ LC Surgeon & & $\begin{array}{l}0.088^{* * *} \\
(0.021)\end{array}$ \\
\hline Physician-Procedure FE & Yes & Yes \\
\hline Half-Year FE & Yes & Yes \\
\hline $\begin{array}{l}\text { Unique Physician- } \\
\text { Procedure Pairs }\end{array}$ & 11,465 & 11,465 \\
\hline Observations $(\mathrm{N})$ & 160,510 & 160,510 \\
\hline
\end{tabular}

Notes: "Splitter" indicates that the physician provides at least some of the relevant procedure cases at both ASC and HOPD settings to their commercially insured patients. "LC Surgeon" is our treatment group (i.e., laparoscopic cholecystectomy surgeons). The control group is composed of all provider-procedure combinations among non-LC physicians observed over our analytic window. During the pre-period, the splitter prevalence rate for LC surgeons and LC commercial cases in our analytic sample is $12 \%$.

** $\mathrm{P}$ value at $0.05 * * * \mathrm{P}$ value at 0.01 , standard errors clustered at the physician level 


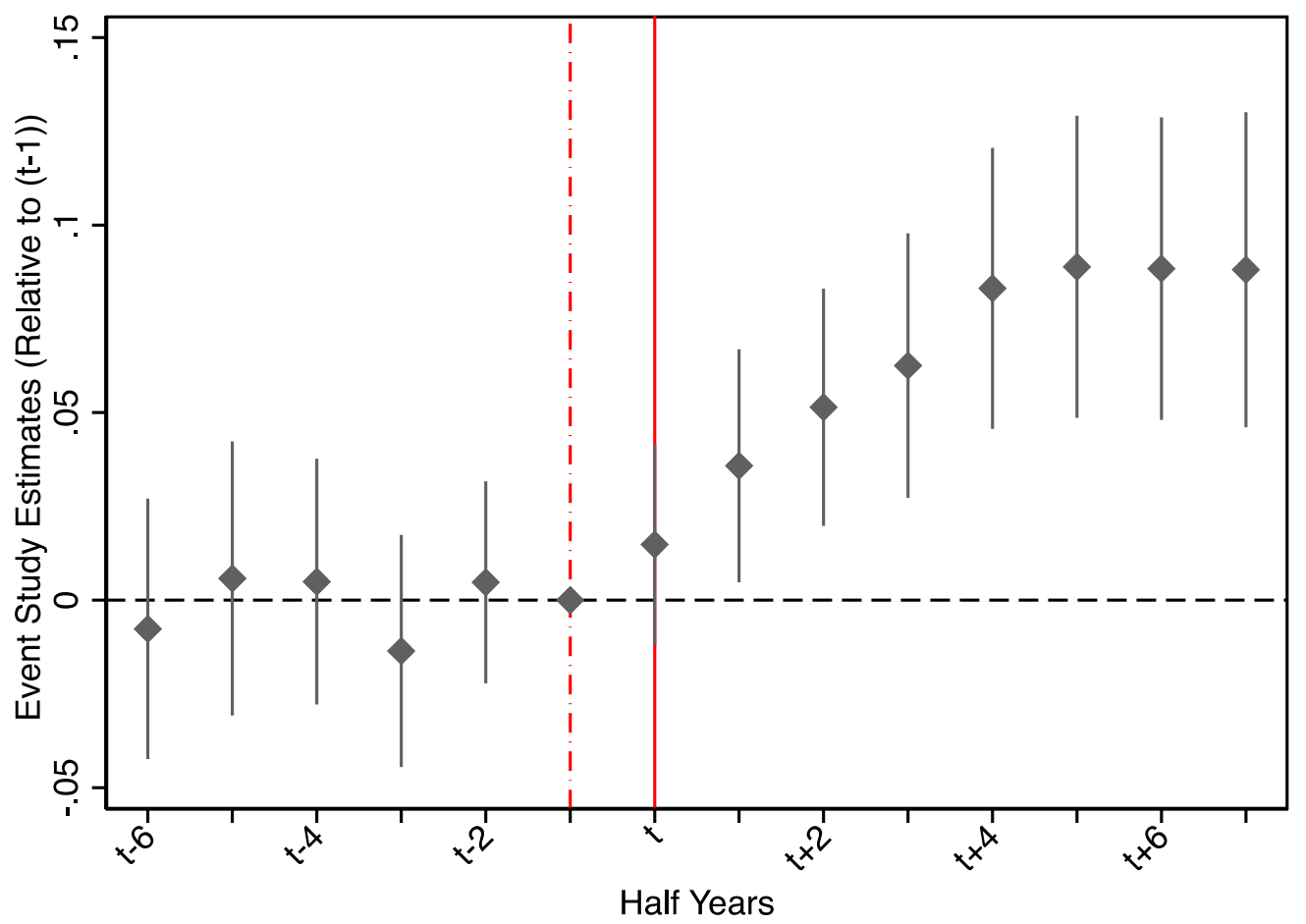

Fig 3. Spillover Effect of Medicare Policy Change for Non-Medicare LC Patients

Notes: The treatment group is all LC surgeons consistently observed over our 2005-2011 period. "Splitter" indicates that the physician provides at least some of the relevant procedure cases at both ASC and HOPD settings. The control group is composed of all provider-procedure combinations among non-LC physicians observed over our analytic window. Time periods are in half-year increments. The variables are constructed based on only commercially insured (i.e., private, non-Medicare payer) cases for the physicians in the sample. $\mathrm{N}=160,510$, unique provider-procedure fixed effects $=11,465$. During the pre-period, the splitter prevalence rate for LC surgeons and LC cases in our analytic sample is $12 \%$. 


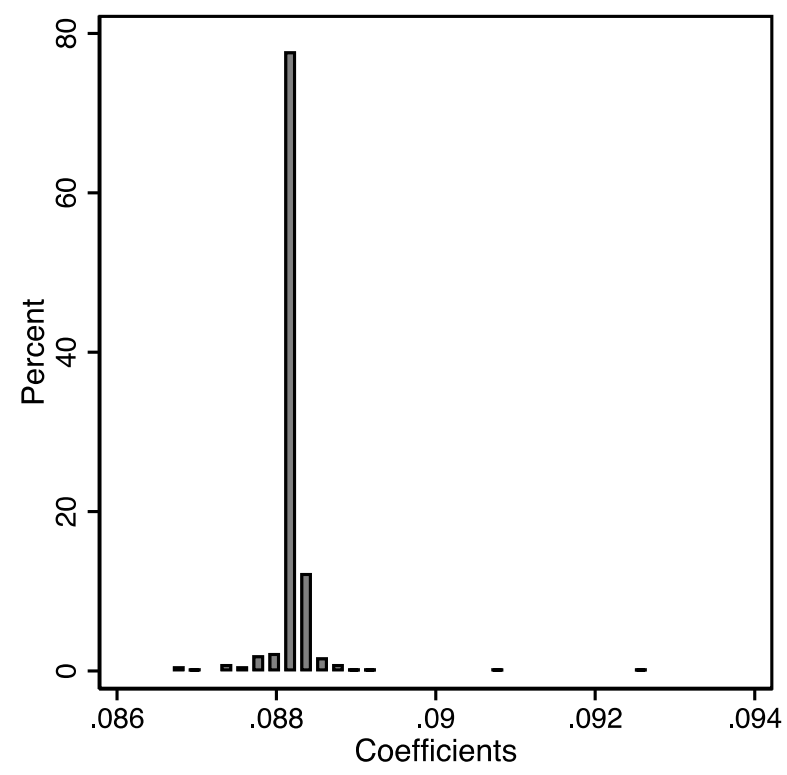

(a) Systematically Excluding One Control Group Per Iteration

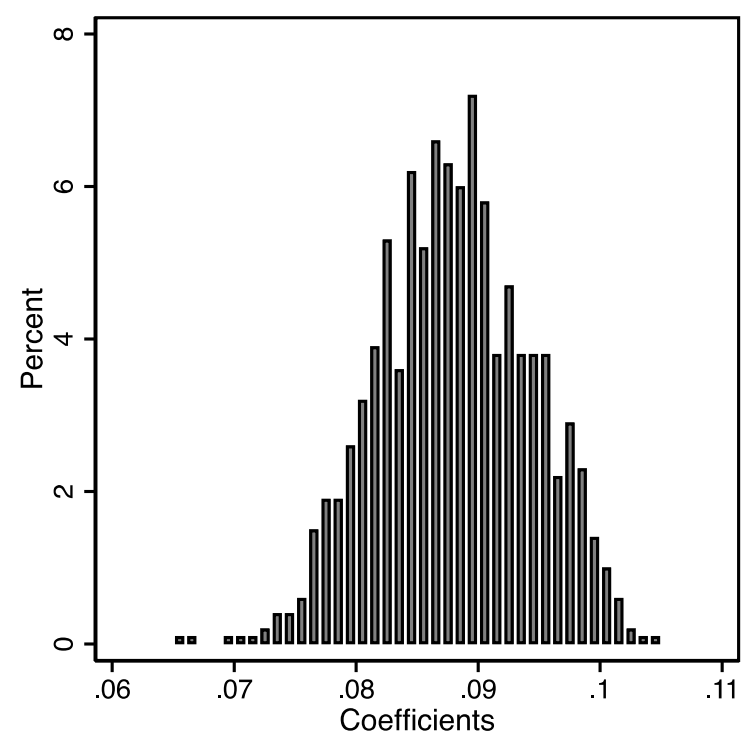

(b) Randomly Drawn Control Groups: 1000 Iterations

Fig 4. Robustness Checks Against Alternative Control Groups for the Direct (Medicare) DD Effect

Notes: Panel A represents 358 re-estimations for the direct effect on LC Surgeons' probability of splitting Medicare LC cases between ASC and HOPD settings. Control group procedures are dropped one-by-one across the iterations. Panel B includes the DD estimates for 1,000 iterations of a randomly drawn 50\% control group of non-LC principle procedures 


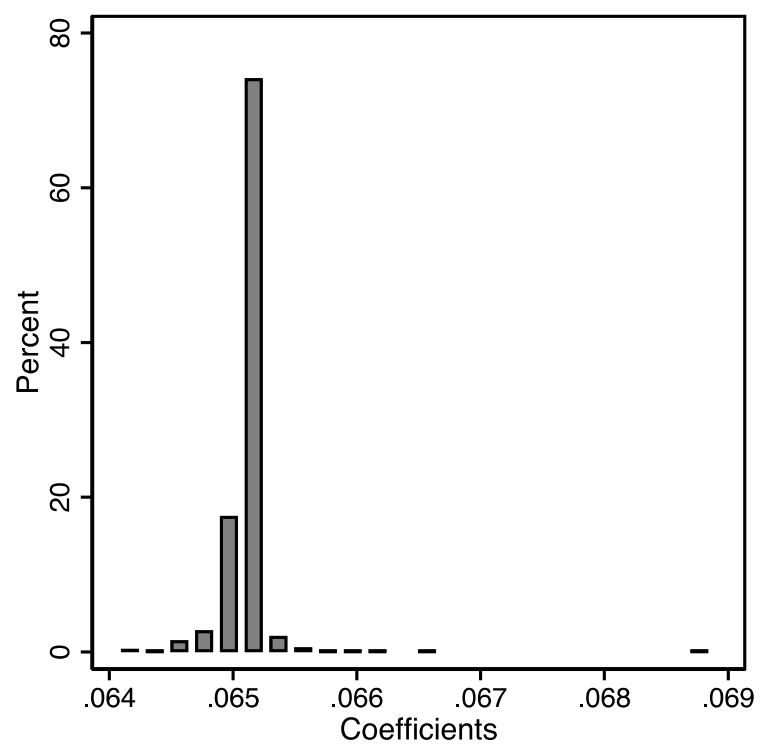

(a) Systematically Excluding One Control Group Per Iteration

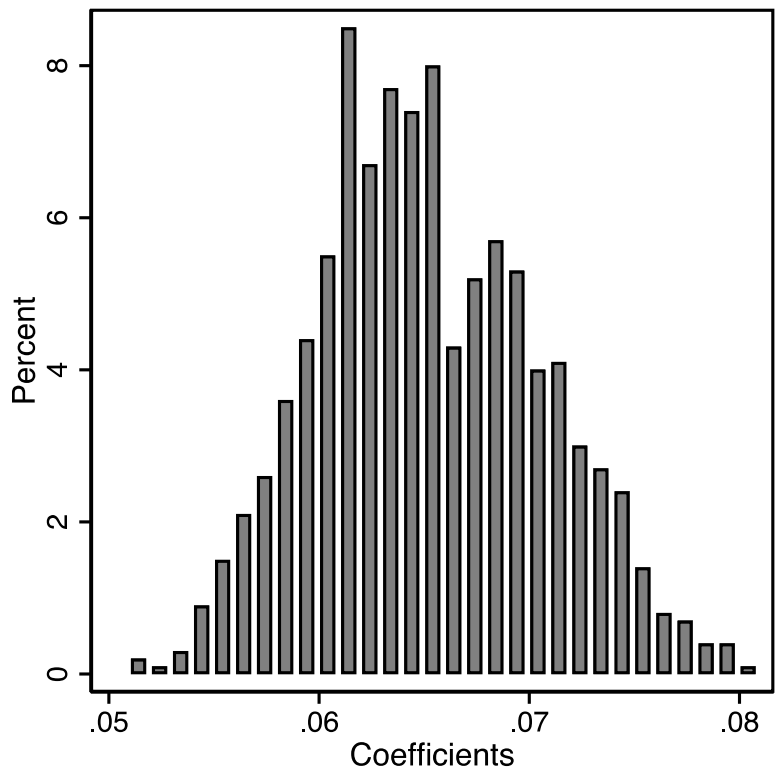

(b) Randomly Drawn Control Groups: 1000 Iterations

Fig 5. Robustness Checks Against Alternative Control Groups for the Spillover (Commercial) DD Effect

Notes: Panel A represent 540 re-estimations for the spillover effect on LC Surgeons' probability of splitting commercial payer LC cases between ASC and HOPD settings. Control group procedures are dropped one-by-one across the iterations. Panel B includes the DD estimates for 1,000 iterations of a randomly drawn $50 \%$ control group of non-LC principle procedures. 


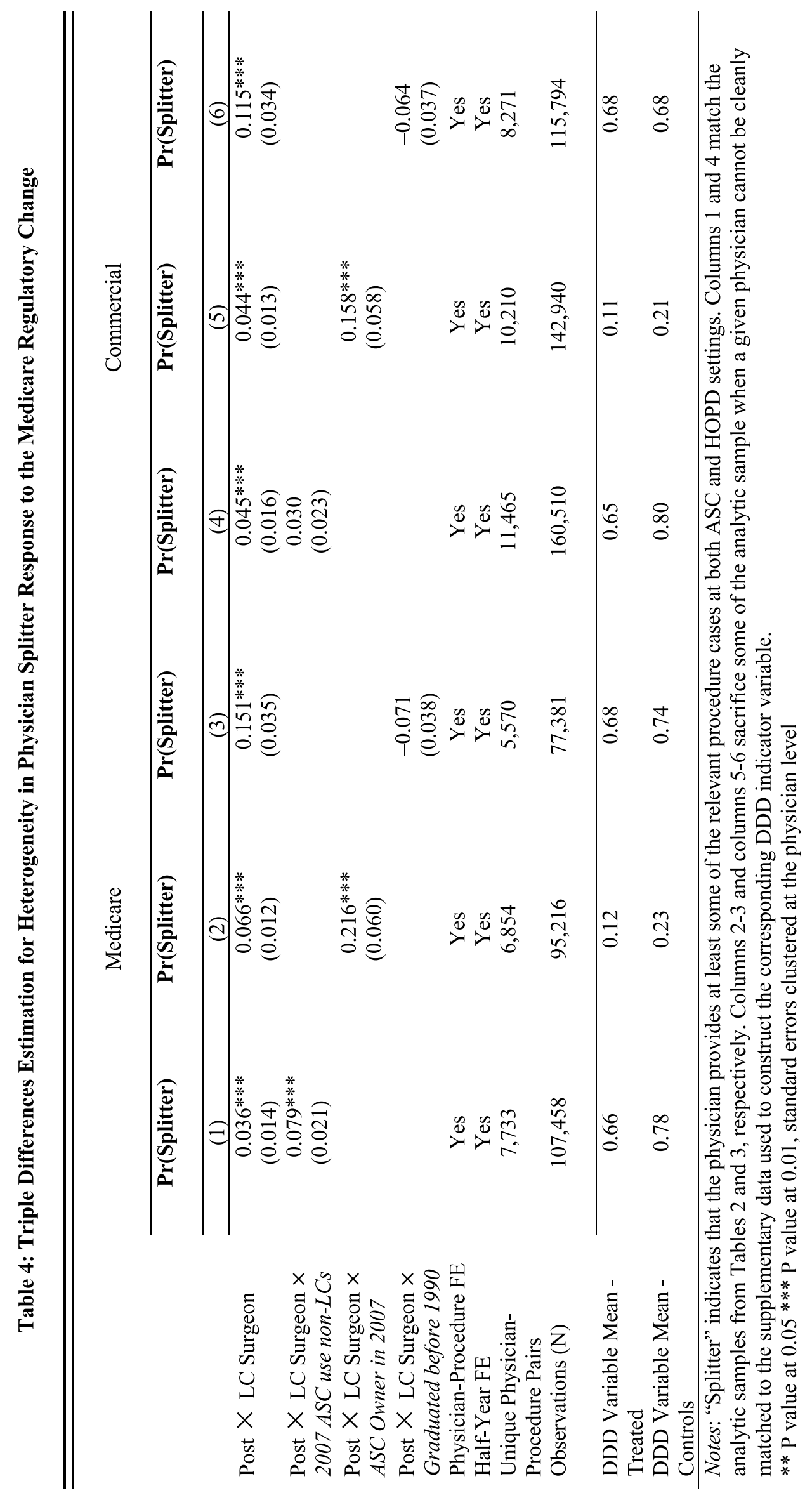


Table 5: Diff-in-Diff Estimates for the Direct and Spillover Effect of Medicare Regulation on the Likelihood of Receiving a Routine Discharge to Home Following the Outpatient Procedure

\begin{tabular}{lcc}
\hline \hline & Medicare & Commercial \\
& Pr(Routine Discharge) & Pr(Routine Discharge) \\
\cline { 2 - 3 } & $(1)$ & $(2)$ \\
\cline { 2 - 3 } Post $\times$ LC Surgeon & -0.0002 & 0.0008 \\
& $(0.0025)$ & $(0.0012)$ \\
Physician-Procedure FE & Yes & Yes \\
Half-Year FE & Yes & Yes \\
Unique Physician- & 7,733 & 11,465 \\
Procedure Pairs & 107,458 & 160,510 \\
Observations (N) & & \\
LC Surgeon Pre-Period & $98.6 \%$ & $99.6 \%$ \\
Mean Percent & & \\
\hline Notes: Analytic samples match Table 2 and Table 3, respectively, for the \\
corresponding payer market. The outcome is equal to one when the patient is \\
discharged home and zero otherwise- meaning a transfer for further care elsewhere. \\
** P value at $0.05 * * *$ P value at 0.01, standard errors clustered at the physician level
\end{tabular}


Table 6: Diff-in-Diff Estimates for Medicare Regulation Spillover Effects on Untargeted (Non-LC) Procedures

\begin{tabular}{lcccc}
\hline \hline & \multicolumn{2}{c}{ Medicare } & \multicolumn{2}{c}{ Commercial } \\
\cline { 2 - 5 } & Pr(Splitter) & $\begin{array}{c}\text { Pr(Use ASC w/ } \\
\text { Lap Tech) }\end{array}$ & Pr(Splitter) & $\begin{array}{c}\text { Pr(Use ASC w/ } \\
\text { Lap Tech) }\end{array}$ \\
\cline { 2 - 5 } & $(1)$ & $(2)$ & $(3)$ & $(4)$ \\
nost $\times$ LC Surgeon & -0.005 & 0.020 & 0.012 & 0.033 \\
Physician-Procedure FE & $(0.018)$ & $(0.025)$ & $(0.015)$ & $(0.019)$ \\
Half-Year FE & Yes & Yes & Yes & Yes \\
Unique Physician- & Yes & Yes & 12,357 & Yes \\
Procedure Pairs & 7,233 & 7,233 & 12,357 \\
Observations (N) & 101,262 & 101,262 & 172,998 & 172,998 \\
\hline
\end{tabular}

Notes: Analytic sample is restricted to physicians present in the main spillover analysis (Table 3) and their non-LC procedures belonging to those physicians that are consistently observed 2005-2011. "Splitter" indicates that the physician provides at least some of the relevant procedure cases at both ASC and HOPD settings. "Use ASC w/ Lap Technology" is equal to one for a physician-half-year when at least one of the relevant non-LC procedures takes place within an ASC that currently has laparoscopic surgery technology. "LC Surgeon" is composed of our treatment group (i.e., laparoscopic cholecystectomy surgeons), just as before.

** $\mathrm{P}$ value at $0.05 * * * \mathrm{P}$ value at 0.01 , standard errors clustered at the physician level 


\section{Appendix A}

\section{Conceptual Model for Public Payer Regulatory Externalities}

In order to guide our empirical investigations, we first model how physicians decide whether to perform a given procedure at an ASC for at least some of their patients, with exclusive use of the HOPD setting serving as the outside option. Surgeons are typically multiproduct firms that supply a variety of services (i.e., procedures) to patients. For example, a general surgeon that performs laparoscopic cholecystectomies also typically performs many other procedures-e.g., surgical excisions, colonoscopies, upper endoscopies, hernia repairs, etc. These surgeons also tend to sell the same service to multiple payers (e.g., Medicare, Medicare Advantage, Medicaid, and nonelderly commercial (private) insurers).

We begin by indexing procedure-by-payer combinations as $j \in J-$ e.g., spinal injections paid by Medicare or colonoscopies paid by commercial insurance. For the purpose of the model (and aligning with the empirical implementation below), we combine all non-Medicare Advantage and non-Medicaid commercial insurance payers into a single payer grouping. ${ }^{1}$

Next, let $R^{j}$ indicate whether the payer-procedure combination is permitted in the ASC setting. More precisely, $R^{j}$ indicates whether the payer will reimburse the facility fee portion of the associated claim if the procedure is performed within an ASC. As previously remarked, Medicare would not pay facility fees for ASC-delivered laparoscopic cholecystectomy (hereafter 'LC') surgeries prior to 2008, which amounted to a de facto ASC ban for this surgical procedure for FFS Medicare patients.

\footnotetext{
${ }^{1}$ Among ASC firms during our study period, the vast majority of cases belong to two payer types: nonelderly commercial and traditional (FFS) Medicare. Other payers, such as Medicaid, receive vanishingly small shares of ASC output. This is also true outside of Florida and across the US-see Hall et al. (2017).
} 
Consider a procedure, like LC surgeries, for which some patients or patient types will always receive care in a HOPD setting, so that a physician's decision to use an ASC for some other patients is equivalent to the decision to divide her caseload across the two treatment settings. We model a physician's discrete choice to perform a procedure at an ASC for at least some of her patients (i.e., behave as a 'splitter') as a function of the reimbursement rule for the payer-procedure combination $\left(R^{j}\right)$, physician preferences, patient preferences, and the physician's extensive margin use of ASC's for other payer-procedure combinations:

$$
A S C_{i}^{j}=f\left(R^{j}, v_{i}^{j},\left\{A S C_{i}^{-j}\right\}\right)
$$

Here, $A S C^{j}$ is an indicator for whether the physician delivers any of her $j$-type services at an ASC, and $i$ indexes individual physicians. The term $v_{i}^{j}$ combines all information on the physician's preferences and her patients' characteristics as these relate to providing service $j$ at an ASC. Examples of such considerations include the distribution of medical appropriateness for ASC use among the physician's patients, the physician's beliefs about patient convenience of ASCs versus HOPDs, idiosyncratic physician treatment preferences, and so on. We do not further decompose or specify this composite term as it will ultimately be held constant (i.e., differenced out) in the quasi-experiment that we exploit below in the empirical analysis.

Importantly, for explaining the possibility of spillovers from Medicare rulemaking onto the commercially insured patient population, we allow $A S C_{i}^{-j}$ - the physician's' decision to use ASCs at all for other payer-procedure combinations (denoted via $-j$ ) - to enter the discrete choice for procedure $j$. This term allows for spillovers across procedure types and/or payers. For example, 
it could be that scheduling a given surgery for commercially insured patients at an ASC becomes more attractive once the physician can schedule Medicare patients for the exact same surgery at an ASC. Such interactions, which depend on the physician's choices over $-j$, are distinct from underlying physician preferences over ASC use for procedure $j$, which is captured in $v_{i}^{j}$.

Because we ultimately investigate what fraction of physicians change behavior by splitting their place of service between ASCs and HOPDs in response to a Medicare policy change, it is useful to aggregate Equation (A1) over the population of physicians. We denote the acrossphysician mean of the ASC indicator function with $\overline{A S C^{j}}$, where the physician subscript $i$ has been removed. This aligns with the empirical exercise below and smooths the discrete choice function, making it differentiable for the purpose of considering comparative statics.

Consider the simple case of just two payer-procedure combinations: $j$ denoting a particular procedure paid for by a commercial insurer, and $-j$ denoting the same procedure paid for by Medicare. Abstracting from binary nature of the ASC ban, and considering a marginal change (such as adjusting rules about what patients are permissible for ASC treatment) for illustration, the impact of changing $R^{-j}$ (the Medicare reimbursement rule) on $\overline{A S C^{j}}$ (the provision of the procedure for commercially insured patients) can be expressed as:

$$
\frac{\partial \overline{A S C^{j}}}{\partial R^{-j}}=\frac{\partial \overline{A S C^{j}}}{\partial \overline{A S C^{-j}}} \times \frac{\partial \overline{A S C^{-j}}}{\partial R^{-j}}
$$

In practice, with an appropriate exogenous shock that manipulates $R^{-j}$, we can estimate $\frac{\partial \overline{A S C^{j}}}{\partial R^{-j}}$ and $\frac{\partial \overline{A S C^{-j}}}{\partial R^{-j}}$, where $j=$ Commercial $\times L C$ and $-j=$ Medicare $\times L C$. We do so below. 
Conditional on these estimates, $\frac{\partial \overline{A S C^{j}}}{\partial \overline{A S C^{-j}}}$ is pinned down by Equation (A2). Recovering $\frac{\partial \overline{A S C^{j}}}{\partial \overline{A S C^{-j}}}$ reveals the extent to which Medicare's regulatory prohibition against ASCs for a certain type of surgery affects the treatment of non-Medicare patients receiving the same procedure. If $\frac{\partial \overline{A S C^{j}}}{\partial \overline{A S C^{-j}}}>0$, then Medicare newly allowing ASC facilities for service delivery crowds in privately insured (commercial) patients. If $\frac{\partial \overline{A S C^{j}}}{\partial \overline{A S C^{-j}}}<0$, the opposite would prove true-i.e., Medicare's regulatory decision ultimately crowds out privately insured (commercial) patients. More broadly, a non-zero result for $\frac{\partial \overline{A S C^{j}}}{\partial \overline{A S C^{-j}}}$ indicates that there are linkages that operate across payers to impact physician decision-making for non-targeted payer-procedure combinations.

As previously remarked, quasi-experimental manipulation of $R^{-j}$ is required to identify such spillover effects because observed values of $A S C_{i}^{j}$ and $A S C_{i}^{-j}$ could be correlated within physicians for many reasons, including physician preferences or sorting of patient types across physicians. Given quasi-experimental manipulation of $R^{-j}$, the key underlying assumption for identifying $\frac{\partial \overline{A S C^{j}}}{\partial \overline{A S C^{-j}}}$ is that there is no direct effect of $R^{-j}$ on $\overline{A S C^{j}}$. We return to this possibility and assess available empirical evidence related to it in Section 7.

Because both payers and procedures vary across the index $j$, Equation (A2) implies the possibility of within-physician spillovers across surgery types (within a payer) as well as across payers (within a surgery type). For example, the expanded ability to schedule a Medicare surgery at an ASC could generate new take-up of ASC use for other Medicare-reimbursed procedure types at ASCs, even without a change in Medicare policy that directly affects these other procedures. 
This means that a narrow Medicare regulatory change targeting LCs could in theory influence physicians' treatment setting decisions for three broad categories of payer-procedure combinations: 1) non-Medicare LCs 2) Medicare non-LCs and/or 3) non-Medicare non-LCs. For the purposes of the model, we have been assuming that each physician performs every $j \in J$ payer-procedure combination, though in what follows, we use physicians who are not exposed to the regulatory change (i.e., who never perform the Medicare policy-targeted LC surgery) as a control group for measuring the size of spillovers. Additionally, the magnitude of $\frac{\partial \overline{A S C^{j}}}{\partial \overline{A S C^{-j}}}$ may vary across the specific $j$ and $-j$ being considered. Our subsequent empirics investigate each of these possibilities and hence the size and reach of the spillover effect from the 2008 Medicare policy change.

Finally, we note that the possibility of $\frac{\partial \overline{A S C^{j}}}{\partial \overline{A S C^{-j}}}$ being nonzero is consistent with a family of explanations for the spillover mechanism. For instance, it subsumes the possibility that the physician's cost of ASC treatment setting scheduling is falling in the variety of procedures available to be performed at ASCs. It also subsumes a kind of "norms hypothesis" for physician behavior, which argues that tangible and cognitive costs can lead physicians to adopt similar treatment styles for different patients-i.e., limit treatment customization for a given medical problem (Newhouse and Marquis 1978; Glied and Graff Zivin 2002; Frank and Zeckhauser 2007). Applied to our context, forces related to physician norms or coordination costs do not affect the choice of treatment (i.e., the procedure to be performed) but instead influence where treatment takes place. 


\section{Appendix B}

Appendix Figure B1: Cross-Sectional Correspondence for Intensive Margin ASC Use Within-Physician and Across Payers

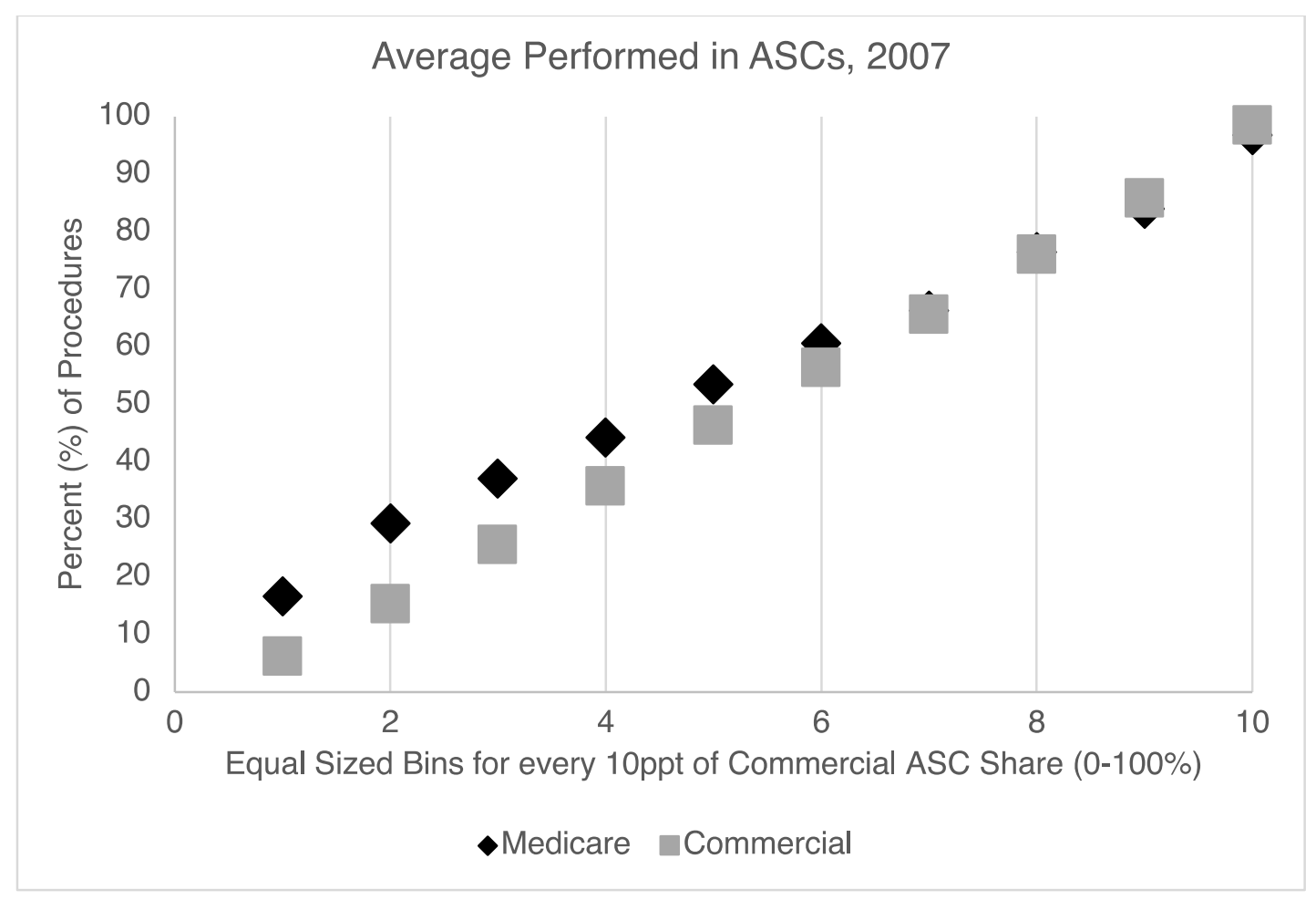

\section{Notes:}

The data are from the universe of ambulatory procedure discharge records in Florida in 2007 and examine the intensive margin correspondence across payers for all procedure-physician combinations. We limit to physicianprocedure pairs with non-zero activity within both treatment settings. We also exclude relative rare procedures for a given physician (i.e., those in the bottom quartile of the volume distribution-less than 14 cases in total). The $\mathrm{x}$-axis consists of 10 equal bins formed by dividing the range of commercial payer ASC shares (0-100\%) into $10 \mathrm{ppt}$ increments. 
Appendix Figure B2: Laparoscopic Cholecystectomy Volumes in Florida 2005-2011 by Payer

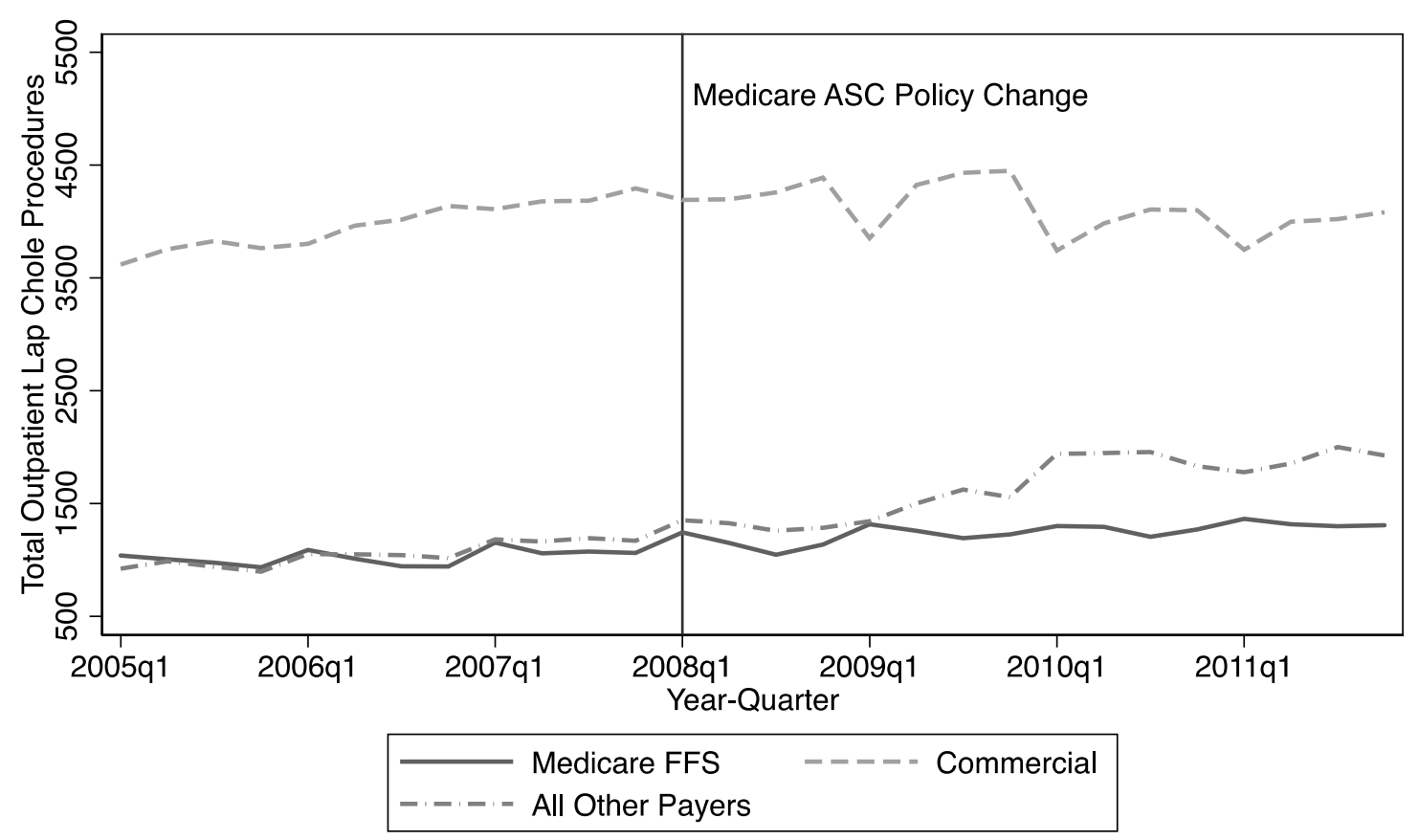

Notes: The data are from the universe of ambulatory procedure discharge records in Florida 2005-2011. The data are at the year-quarter level. 
Appendix Figure B3: Separate Event Study Estimates for the Control Group and Treatment Group by Payer
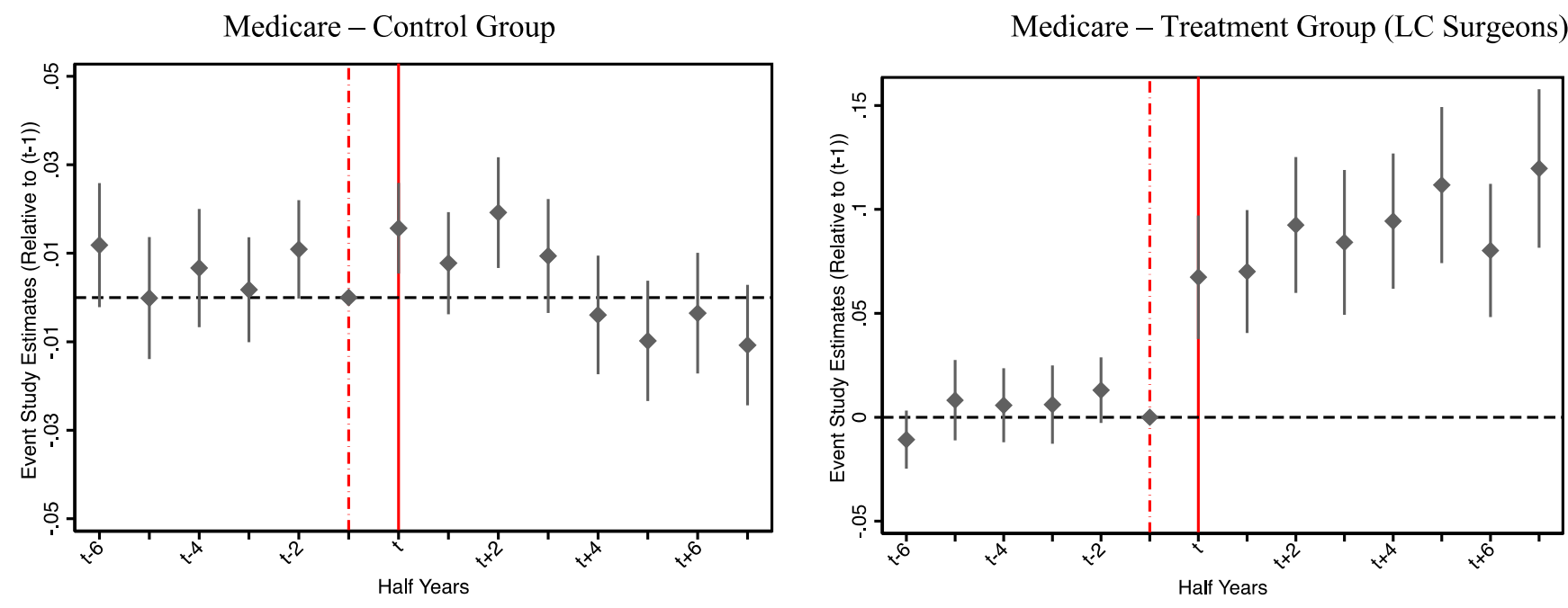

Commercial - Control Group

Commercial - Treatment Group (LC Surgeons)
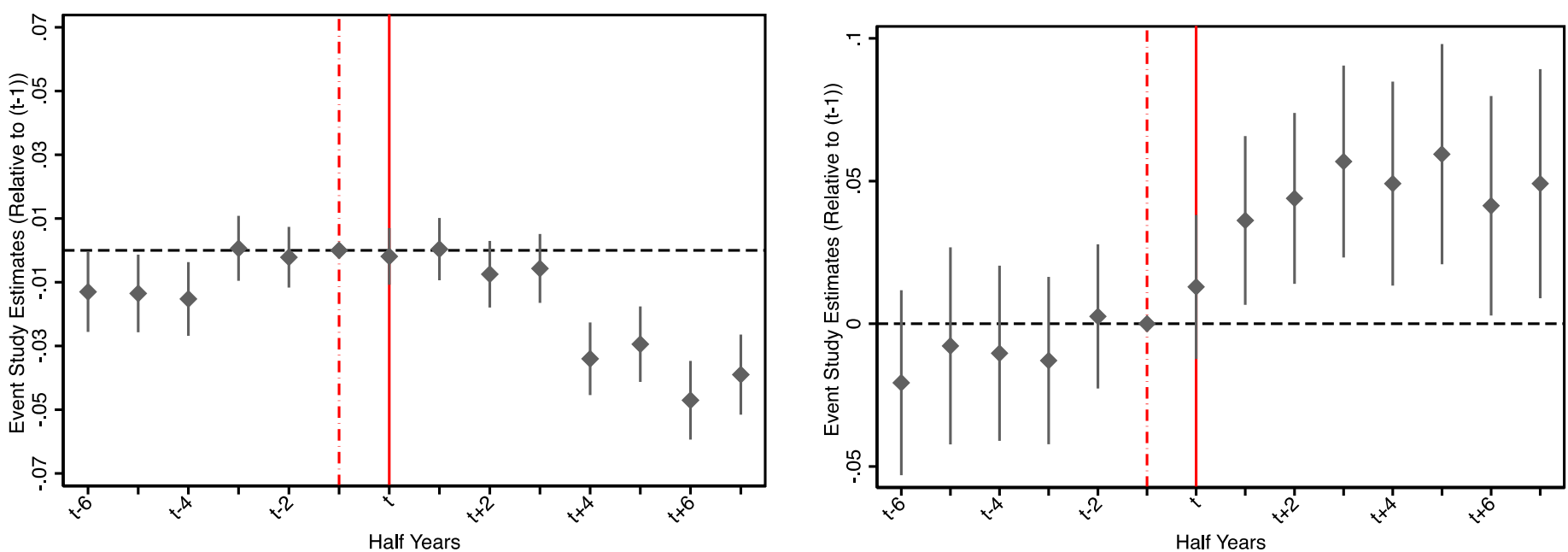

Notes: Control group corresponds to that used in the main event studies displayed in Figures 2 and 3. Of note, the top right panel (Medicare LC Surgeons) is not a precise zero for the pre-period coefficients due to a very small number of LC procedures reported as taking place within an ASC and for a Medicare patient. This may reflect misclassification of these cases or, at least in theory, could reflect a small minority of LC surgeons receiving special permission to perform select Medicare LC cases in the ASC - even though the ASC will receive no subsequent payment from Medicare. One such scenario could be an LC surgeon with a significant ownership stake in the ASC and therefore extensive discretion in such a decision. 
Appendix Table B1: Diff-in-Diff Estimates for the Direct Effect of Medicare Regulation on the Likelihood of Using Both ASC and HOPD Treatment Settings for Medicare Patients

Imposing Balanced LC Surgeon Panel for Medicare Cases

\section{$\operatorname{Pr}($ Splitter)}

\section{DD Estimate}

Physician-Procedure FE

Half-Year FE

Unique Physician-Procedure Pairs

Observations $(\mathrm{N})$

\section{$0.108^{* * *}$}

$(0.020)$

Yes

Yes

7,509

105,126

Notes: "Splitter" indicates that the physician provides at least some of the relevant procedure cases at both ASC and HOPD settings to their Medicare patients. "LC Surgeon" is our treatment group (i.e., laparoscopic cholecystectomy surgeons). The control group is composed of all provider-procedure combinations among non-LC physicians observed over our analytic window.

** $\mathrm{P}$ value at $0.05 * * * \mathrm{P}$ value at 0.01 , standard errors clustered at the physician level 
Appendix Figure B4: Direct Effect of Medicare Policy Change That Allowed LC Surgeries in ASC Settings When Imposing a Balanced Panel of Medicare LC Cases for the Treatment Group

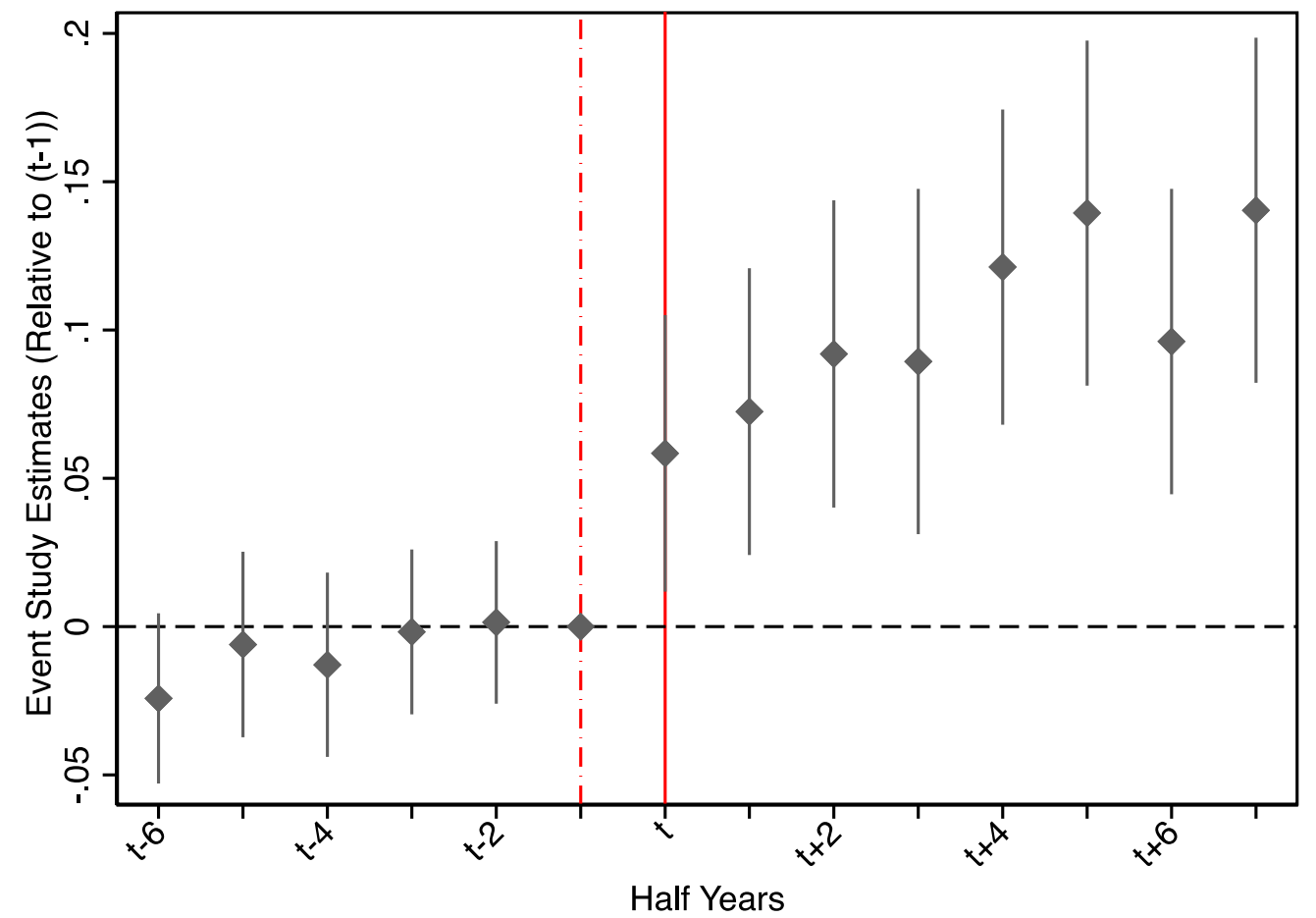

Notes: The treatment group is all LC surgeons consistently observed over our 2005-2011 period in the commercial market. "Splitter" indicates that the physician provides at least some of the relevant procedure cases at both ASC and HOPD settings. The control group is composed of all provider-procedure combinations among non-LC physicians observed over our analytic window. Time periods are in half-year increments. The variables are constructed based only on traditional Medicare (i.e., fee-for-service) cases for the physicians in the sample. $\mathrm{N}=107,458$, unique provider-procedure fixed effects $=7,733$. 


\section{Appendix Figure B5: Sample Size Changes When Systematically Re-Estimating the DD Model Without One Control Group Principal Procedure}

(a) Medicare

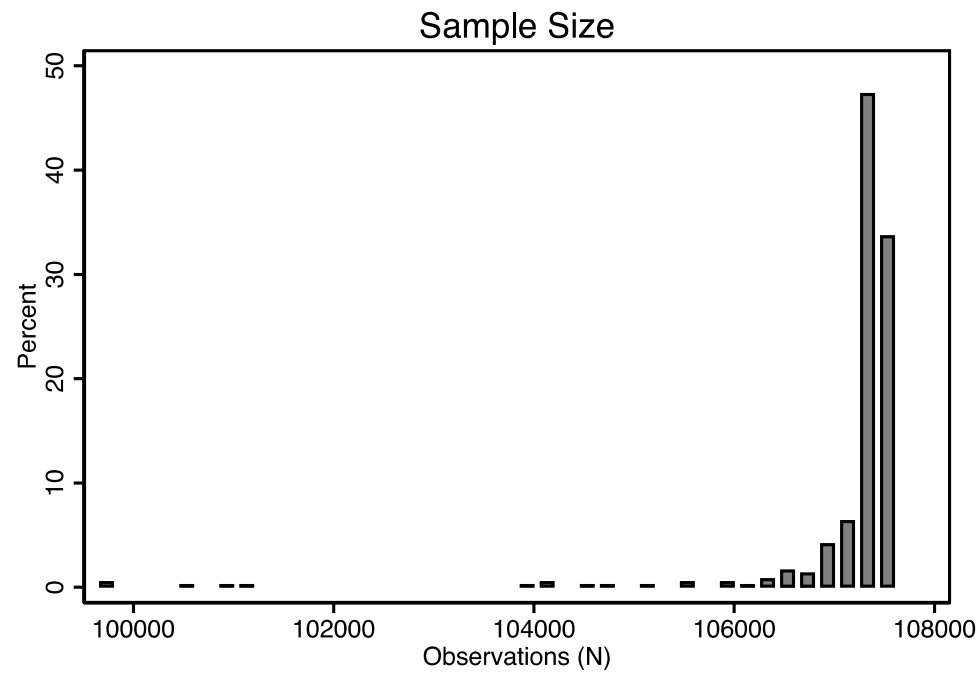

(b) Commercial

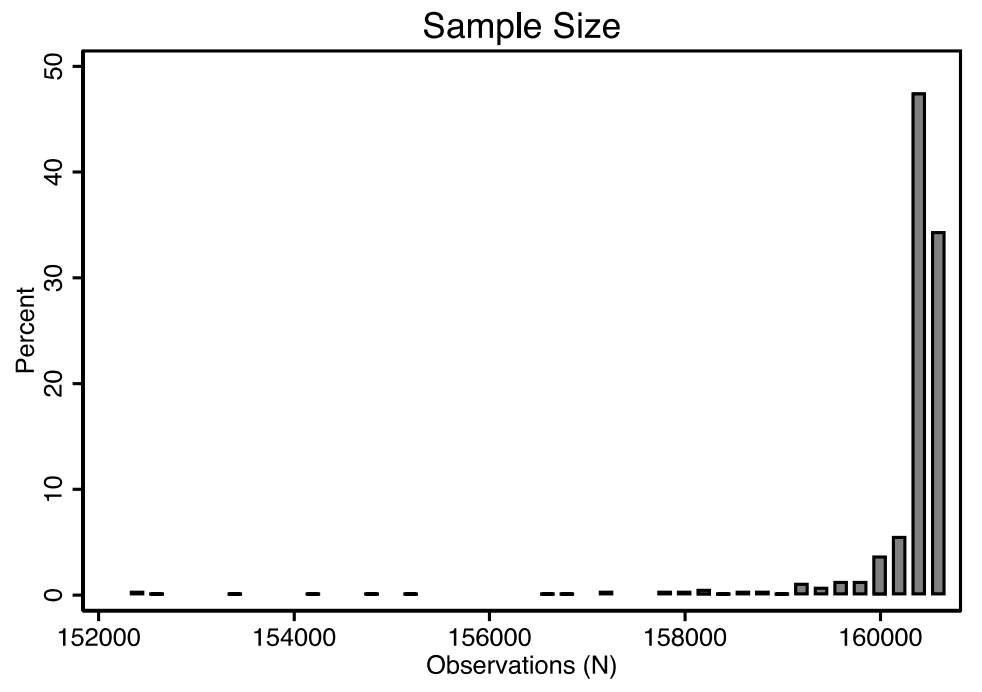




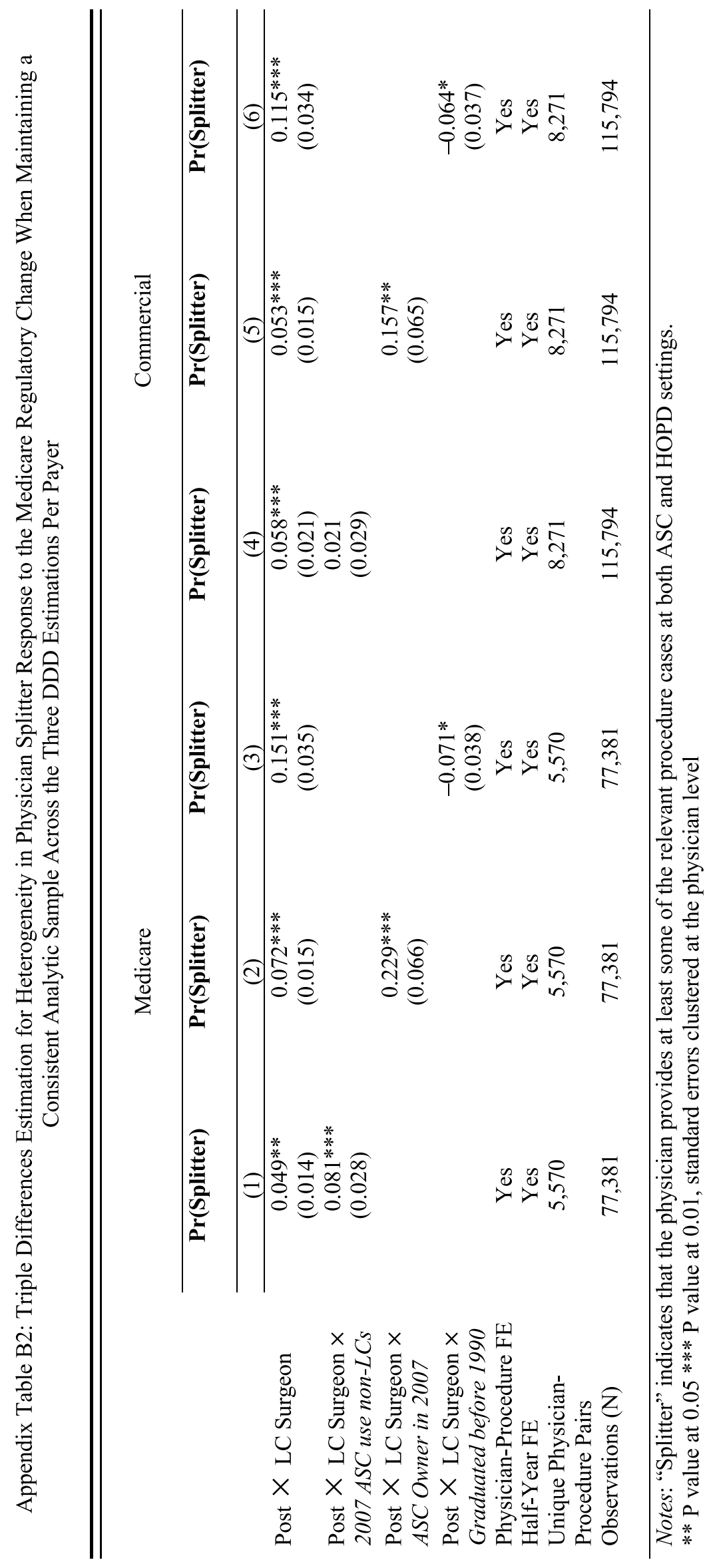


Aggregating All Non-LC Procedures Per Physician

We maintain the same treatment-control designations of physicians as before, and we estimate specifications that parallel Equations (1) and (2) in structure and notation:

$$
\begin{gathered}
Y_{i t}=\zeta \text { Post }_{i}+\delta\left(\mathbf{1}\left(\text { Post }_{t}\right) \times \mathbf{1}\left(\text { LCSurgeon }_{i}\right)\right)+\lambda_{i}+\varepsilon_{i t} \\
Y_{i t}=\sum_{\substack{m=-6 \\
m \neq-1}}^{7} \gamma_{m}(\mathbf{1}(t+m))+\sum_{\substack{n=-6 \\
n \neq-1}}^{7} \delta_{n}\left(\mathbf{1}\left(\text { LCSurgeon }_{i}\right) \times \mathbf{1}(t+n)\right)+\lambda_{i}+\varepsilon_{i t}
\end{gathered}
$$

The key difference here is that rather than examining the LC surgeries of LC physicians and excluding their non-LC procedures, we examine their non-LC procedures and exclude their LC procedures. The sample restriction in terms of physicians is exactly the same as above (i.e., the main spillover analysis in Table 3). To construct the dependent variable, we group together all non-LC procedures within a physician. If a given physician $(i)$ in a given half-year $(t)$ has a positive case volume for the relevant payer's non-LC procedures within both ASC and HOPD settings, then the physician-by-time period observation is coded as a one and is zero otherwise. Given this construction, observations are at the physician-by-time level, and we use physician fixed effects $\left(\lambda_{i}\right)$ rather than procedure-physician pair fixed effects $\left(\theta_{p}\right)$. Standard errors are clustered at the physician level, and regressions are run separately for the two payer types. 
Appendix Table B3: Diff-in-Diff Estimates for Medicare Regulation Spillover Effects on Untargeted (Non-LC) Procedures When Aggregating all Non-LC Procedures Together at the Physician-Time Level

\begin{tabular}{|c|c|c|c|c|}
\hline & \multicolumn{2}{|c|}{$\operatorname{Pr}($ Splitter) } & \multicolumn{2}{|c|}{$\operatorname{Pr}$ (Use ASC with Lap Technology) } \\
\hline & Medicare & Commercial & Medicare & Commercial \\
\hline & $(1)$ & $(2)$ & (3) & $(4)$ \\
\hline Post $\times$ LC Surgeon & $\begin{array}{l}-0.008 \\
(0.018)\end{array}$ & $\begin{array}{l}-0.002 \\
(0.018)\end{array}$ & $\begin{array}{l}-0.008 \\
(0.019)\end{array}$ & $\begin{array}{l}-0.008 \\
(0.019)\end{array}$ \\
\hline Physician FE & Yes & Yes & Yes & Yes \\
\hline Unique Physicians & 4,040 & 4,112 & 4,040 & 4,112 \\
\hline Observations $(\mathrm{N})$ & 52,599 & 57,567 & 52,599 & 57,567 \\
\hline \multicolumn{4}{|l|}{ LC surgeon } & 0.49 \\
\hline \multicolumn{5}{|c|}{$\begin{array}{l}\text { Notes: Analytic sample is restricted to physicians present in the main spillover analysis (Table } 3 \text { ) and all non-LC } \\
\text { procedures belonging to those physicians. "Splitter" indicates that the physician provides at least some of the } \\
\text { relevant procedure cases at both ASC and HOPD settings. "Use of ASC with Lap Technology" is equal to one for } \\
\text { a physician-half-year when at least one non-LC procedure takes place within an ASC that currently has laparoscopic } \\
\text { surgery technology. "LC Surgeon" is composed of our treatment group (i.e., laparoscopic cholecystectomy } \\
\text { surgeons), just as before. } \\
* * \text { P value at } 0.05 * * * \text { P value at } 0.01 \text {, standard errors clustered at the physician level }\end{array}$} \\
\hline
\end{tabular}


Descriptive Explorations According to Physician Policy Response Type

We also produce a more nuanced but necessarily descriptive set of facts for the same outcomes examined in Appendix Table B3. Specifically, we plot trends in the two outcomes (per payer type) for three mutually exclusive subsets of the treatment group surgeons: those who began splitting commercial LC procedures before the Medicare policy change, those who began doing so only after the policy change, and those who never adopt the splitter style for commercial LC patients during our sample period. The "never" group captures the largest share $(60 \%)$ of our treatment group physicians in the data. The remaining $40 \%$ is nearly evenly divided between the other two classifications. Having this mutually exclusive categorization of LC surgeons subsequently allows us to narrow our attention to the minority of these physicians who plausibly changed their behavior due to the policy.

With these definitions, we can ask whether the physicians who appear to have responded to the Medicare policy in terms of ASC use for commercial LCs also respond in other ways. Examining the behavior of this subgroup more precisely may be informative but requires more subtle attention to interpretation because we are essentially conditioning on the dependent variable from our primary spillover analysis.

Appendix Figures B6 and B7 plot the trends in the outcomes reported in Appendix Table B3for each of these three physician subgroups according to payer type. In each panel of Figure B6, surgeons splitting their commercial LC provision in the pre-period have high rates of doing likewise for their non-LC procedures, and this remains level across the 2008 policy change. Similarly, surgeons who never split their commercial LCs consistently tend to not do so for other (non-LC) commercial and Medicare procedures. Unremarkable trends (with similar level differences) are also seen for these two treated physician subgroups when examining their 
propensity to have a non-zero amount of non-LC procedures performed in ASCs equipped with laparoscopic surgical technology (Figure B7).

The more interesting patterns in Figures B6 and B7 belong to the subgroup of surgeons that are newly using both HOPD and ASC treatment settings for their commercial LC patients once the Medicare deregulation event has taken place. Their probability of using both ASCs and HOPDs for non-LC procedures increases over time, with the most dramatic increases following the Medicare rule change (Figure B6). A similar trend change is evident for the proportion using ASCs with laparoscopic technology for their non-LC procedures (Figure B7). These descriptive patterns could be because the rule change altered their decision making on treatment setting for non-LC procedures as a secondary spillover effect, or it could be because the physicians who ultimately responded to the rule change (reflected in our causal estimates in Tables 2 and 3) were disproportionately drawn from physicians who were closer to the threshold for initiating ASC use for many of their services. It is not possible to distinguish between explanations in these data, but it does raise the possibility of more diffuse spillovers beyond the procedure narrowly targeted by Medicare's regulatory change. ${ }^{2}$ Future work (and different data) would be needed to assess these more diffuse spillovers or to analyze the detailed channels by which physicians' equilibrium behavior changed.

\footnotetext{
${ }^{2}$ For example, it is possible that a regulatory shock that applied to a more common procedure and/or a larger share of physicians could generate a detectable effect along these lines-something future, related research may wish to consider.
} 
Appendix Figure B6: Trends in Splitting for Non-LC Procedures among LC Surgeons by Payer

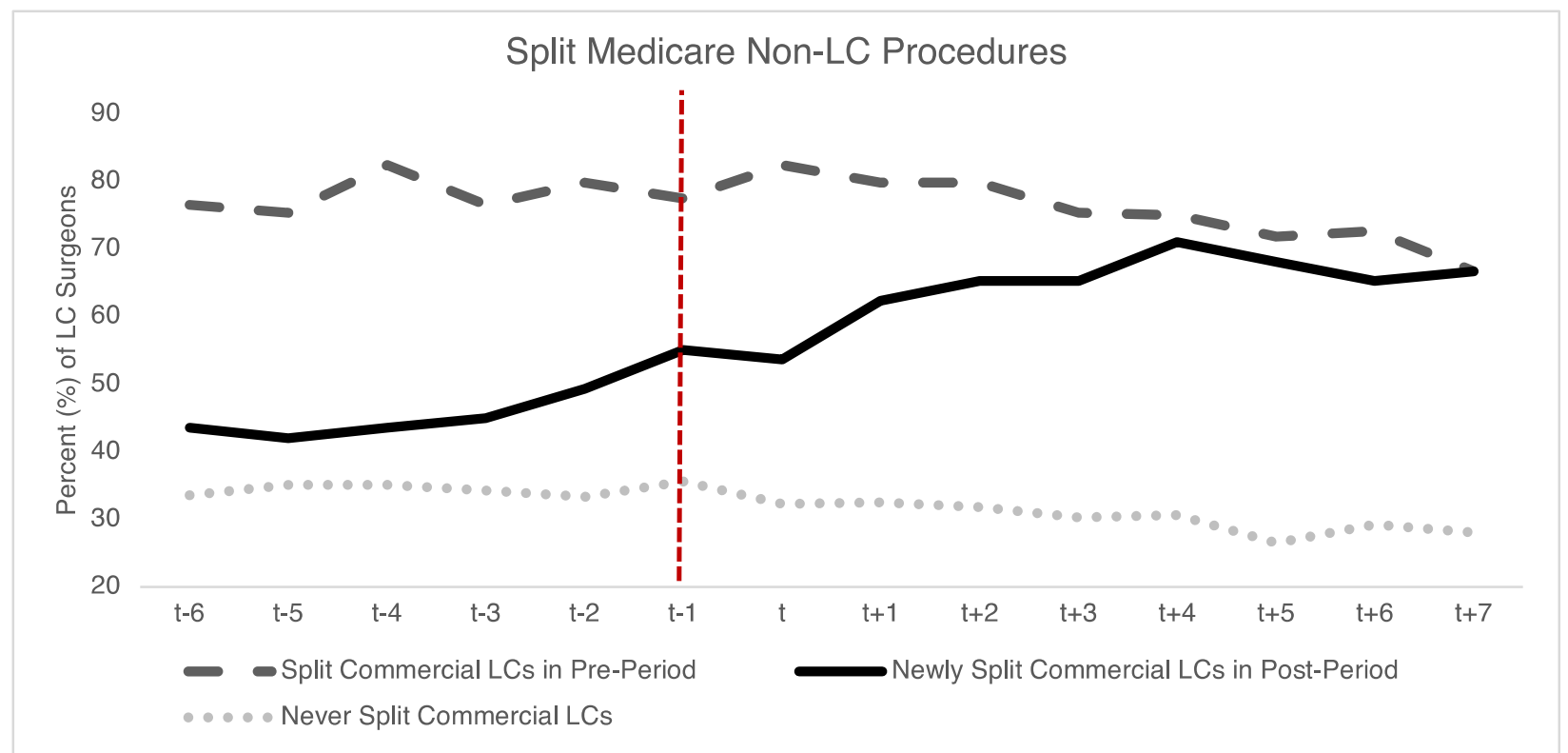

(a) Medicare Outcomes

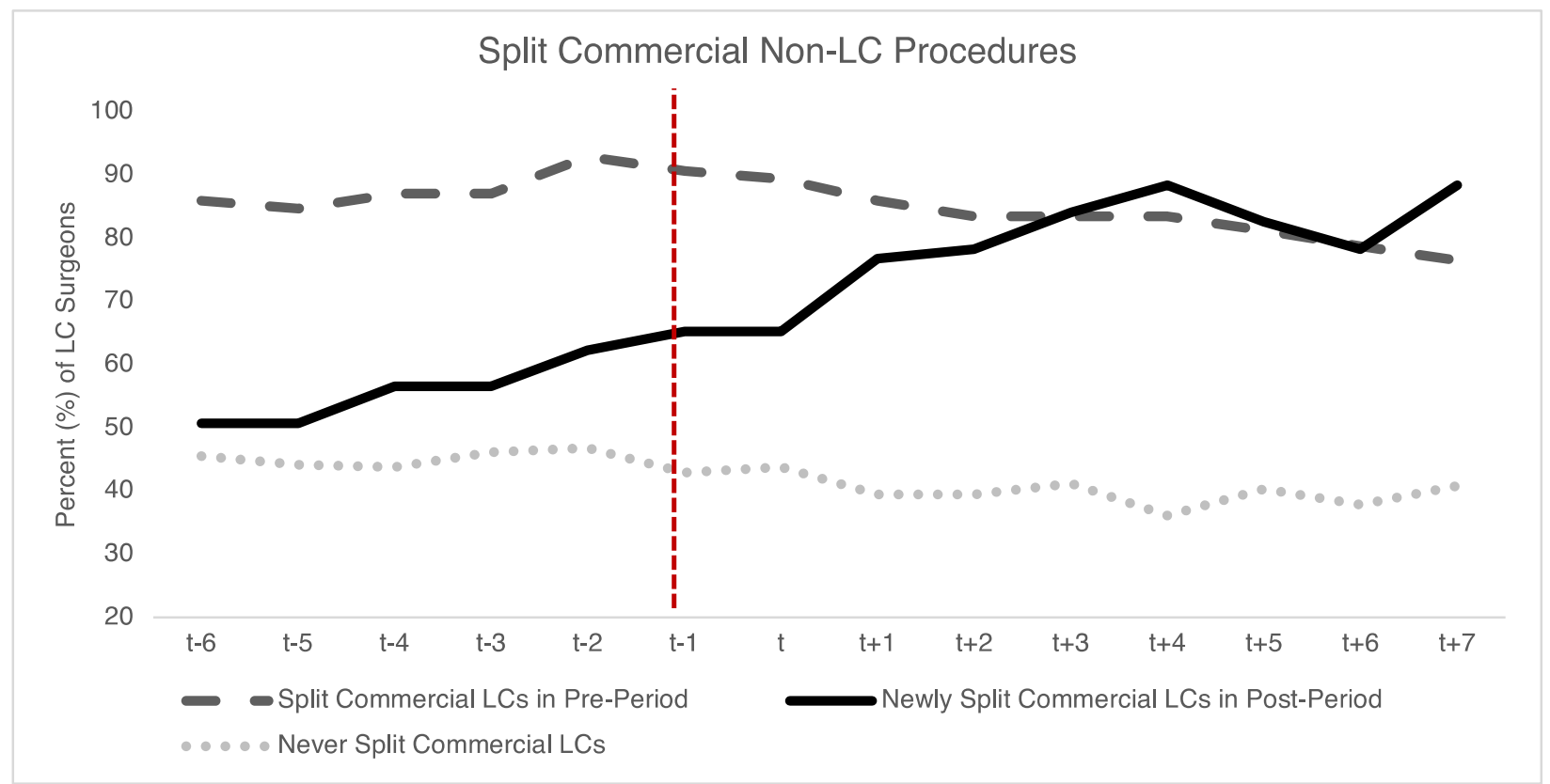

(b) Commercially Insured Outcomes

Notes: Restricts to LC surgeons belonging to our main analytic sample underlying Table 3 and their corresponding non-LC outpatient procedures (i.e., all other services these surgeons provide to the Medicare or commercial market in a given time period). LC surgeons are further stratified by their regulatory response type from the main spillover analysis (Table 3). 
Appendix Figure B7: Trends in Extensive Margin Use of ASCs with Lap Technology for Non-LC Procedures among LC Surgeons by Payer

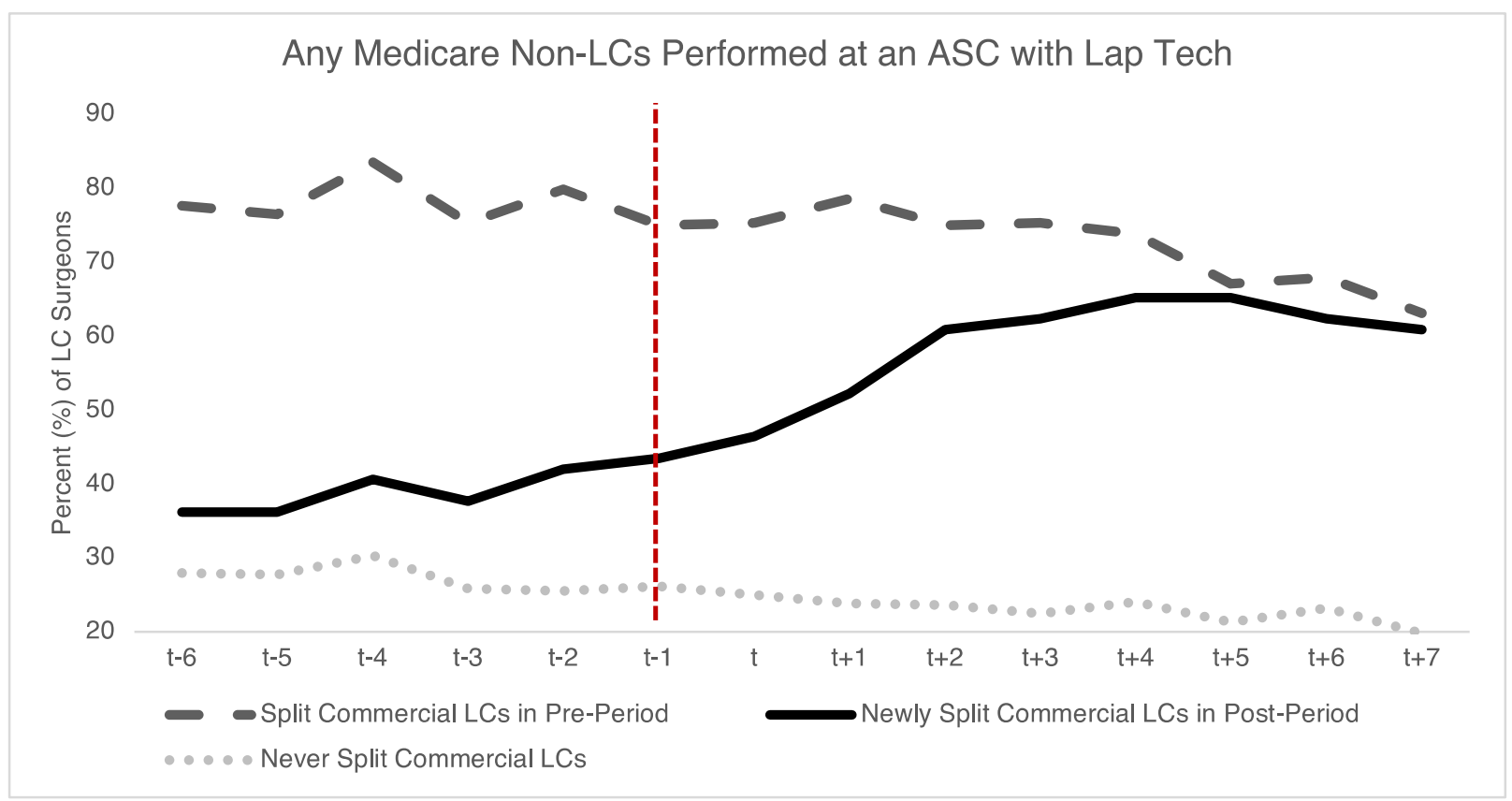

(a) Medicare Outcomes

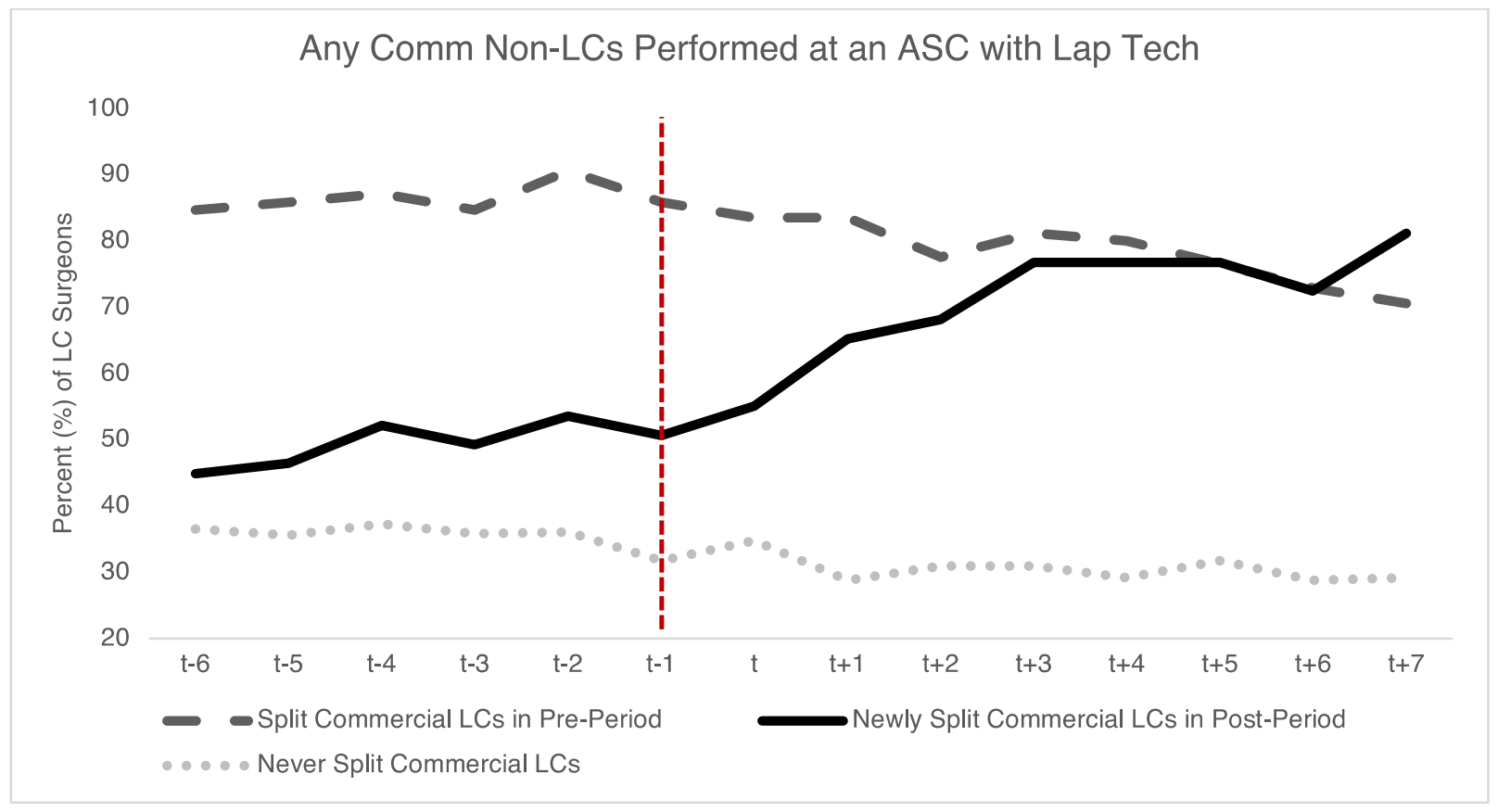

(b) Commercially Insured Outcomes

Notes: Restricts to LC surgeons belonging to our main analytic sample underlying Table 3 and their corresponding non-LC outpatient procedures (i.e., all other services these surgeons provide to the Medicare or commercial market in a given time period). LC surgeons are further stratified by their regulatory response type from the main spillover analysis (Table 3). 
Appendix Table B4: Pre and Post Summary Measures for the Treatment and Control Groups Belonging to our DD Estimation

\begin{tabular}{lcc}
\hline & Pre-Period (2005-2007) & Post-Period (2008-2011) \\
\hline Splitter - Medicare LC Surgeons & -- & $9.8 \%$ \\
Splitter - Medicare Control Group & $29.5 \%$ & $29.3 \%$ \\
$\%$ in ASCs - Medicare LC Surgeons & -- & $5.2 \%$ \\
$\%$ in ASCs - Medicare Control Group & $57 \%$ & $62 \%$ \\
Any ASC Use - Medicare LC Surgeons & -- & $11.0 \%$ \\
Any ASC Use - Medicare Control Group & $66.4 \%$ & $70.5 \%$ \\
Splitter - Commercial LC Surgeons & $12.3 \%$ & $17.5 \%$ \\
Splitter - Commercial Control Group & $38.4 \%$ & $37.1 \%$ \\
& & \\
$\%$ in ASCs - Commercial LC Surgeons & $5.2 \%$ & $8.2 \%$ \\
$\%$ in ASCs - Commercial Control Group & $54.3 \%$ & $59.4 \%$ \\
& & \\
Any ASC Use - Commercial LC Surgeons & $12.9 \%$ & $18.5 \%$ \\
Any ASC Use - Commercial Control Group & $66.6 \%$ & $70.2 \%$ \\
\end{tabular}


Appendix Figure B8: Event Study Estimates for the Medicare Regulation Intensive Margin Effect: Percent of Procedures Performed in ASCs (inverse hyperbolic sine transformation)

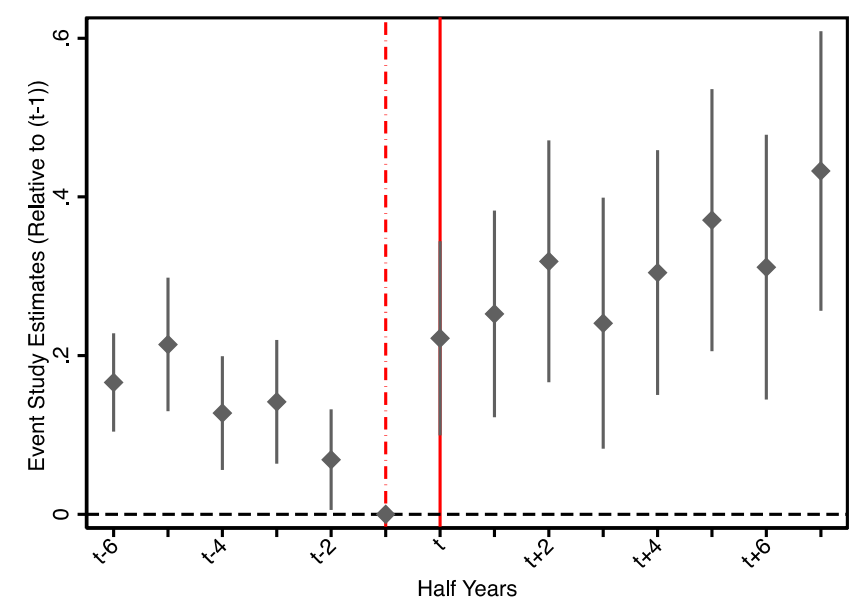

(a) Medicare

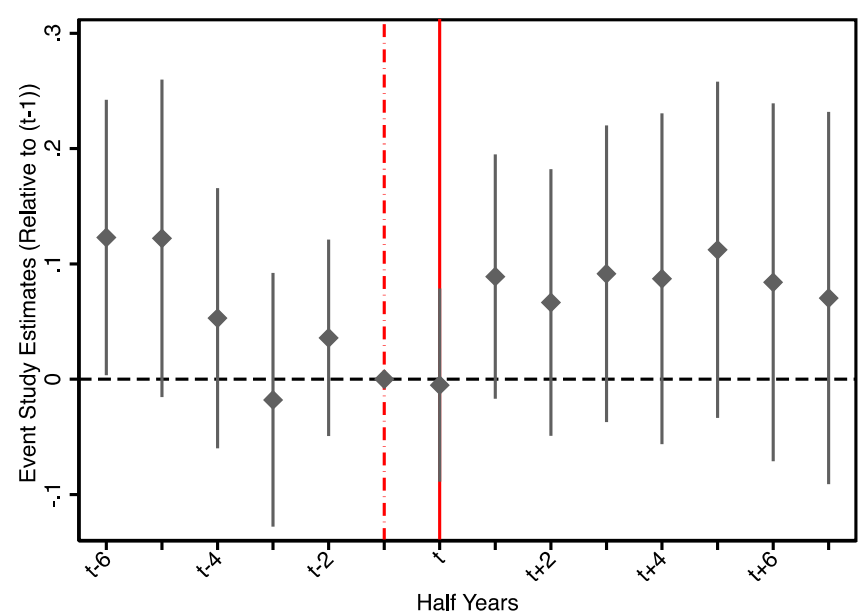

(b) Commercial

Notes: Treatment and control setup mirrors the main analyses. 
Appendix Figure B9: Event Study Estimates for the Medicare Regulation Intensive Margin Effect: Percent of Procedures Performed in ASCs (outcome defined in levels)

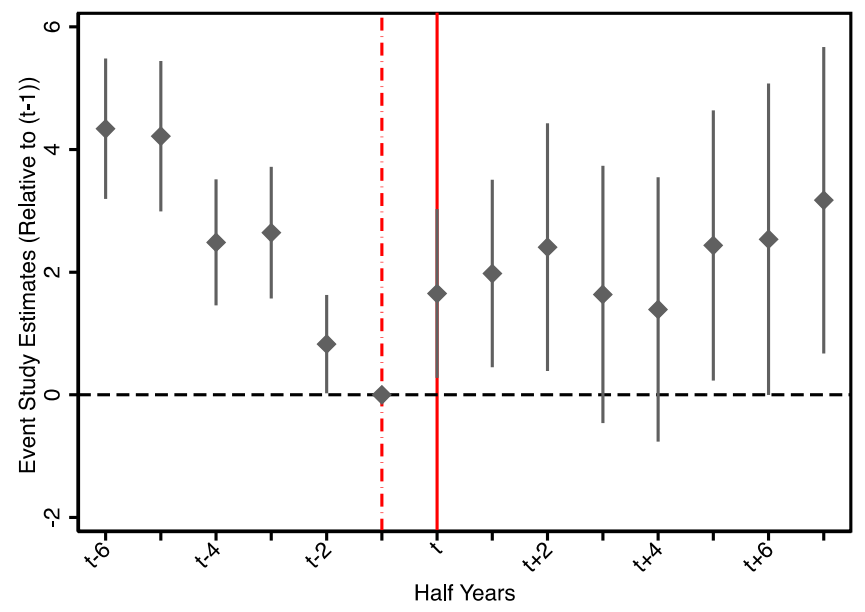

(a) Medicare

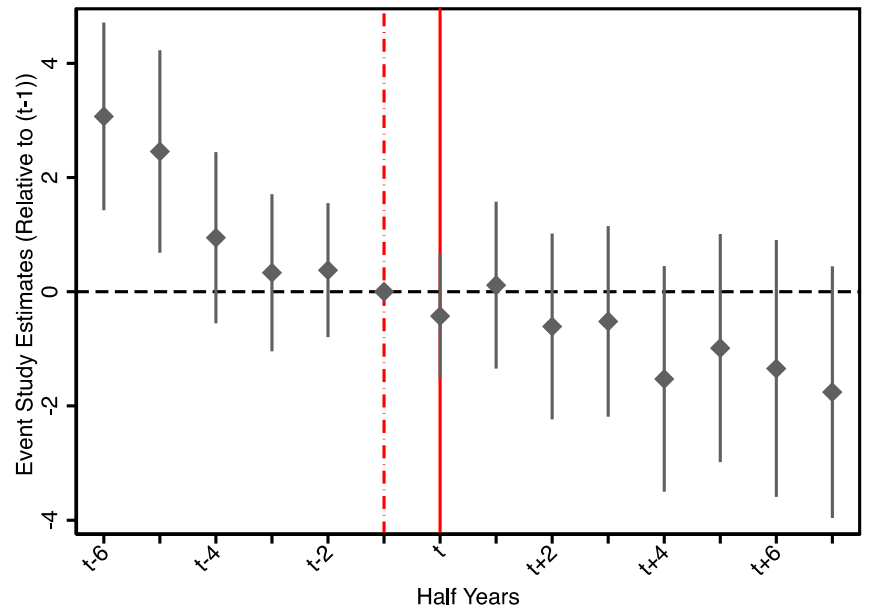

(b) Commercial

Notes: Treatment and control setup mirrors the main analyses. 


\section{Appendix C}

\section{Evidence on Rule Following}

Here we investigate whether commercial insurers adopt Medicare's rules for banning (and lifting the ban) on LCs performed within ASC treatment settings. That said, we cannot observe commercial contract details with LC surgeons - and hence any direct rule following - in our discharge data belonging to our main analyses, which does not include information about reimbursement rules belonging to the various payers. We therefore supplement our existing analyses with Marketscan data from 2005-2012 in order to explore the rule-following mechanism further.

The Marketscan claims database offers the highest quality large, transaction-level, commercial insurer dataset for our time period of interest. Nonetheless, there are several limitations. First, it is impossible to link providers (such as LC surgeons) across procedures and over time in the database. Second, Marketscan is national, not limited to Florida, and lacks geographic identifiers that could allow us to narrow it to align with our main sample. Third, we are unable to track specific facilities in the database and have no geographic information on the location where a given physician service took place. Data contributors (typically large, self-insured firms) also change over time, which creates further ambiguity for the underlying providers and geographic locations represented in the database. For these reasons, the data cannot be used to replicate the same analyses from the paper, which would require a stable sample over time, as well as the ability to track physicians over time. However, these data are well-suited for tracking whether data contributors (e.g., large employers) change their use of ASCs for LC procedures, particularly along the extensive margin (i.e., the margin most consistent with a rule-following effect, should one exist). In other words, once Medicare allows LCs at ASC, do commercial insurers make the same change? We provide such statistics insofar as they can be informative for aggregate patterns and the associated plausibility of a rule-following mechanism.

To investigate whether patterns in the Marketscan data are consistent with rule-following, we aggregate to the level of the payer. In this database this corresponds to the data contributor, and we limit to data contributors consistently present in the data over the study period. We plot the fraction of data contributors for which ASC use is precisely zero in each year. An observation is therefore a payer-year. A decrease in this statistic after 2008 (i.e., fewer commercial payers with 
no LCs performed within ASCs) would be consistent with payers changing their rules in the wake of the Medicare rule change targeting LCs. Panel (a) of Appendix Figure C1 shows no obvious decline following Medicare's deregulation event. Because having exactly zero ASC LC procedures in the claims records may be too stringent of a proxy disallowing ASC use, we also repeat the analysis with a threshold set at $<5 \%$ ASC using in panel (b) of Appendix Figure C1. Again, there is no compelling pattern to suggest a sharp coverage policy change for these commercial payers. Thus, we fail to find clear empirical evidence supporting a rule-following interpretation, though we cannot rule it out. In sum, we are limited in the ability to offer definitive evidence on mechanisms, leaving these questions for future research. 
Appendix Figure C1: Trends in Extensive Margin Use for LC Procedures at the Payer Level within the Marketscan Database, 2005-2012

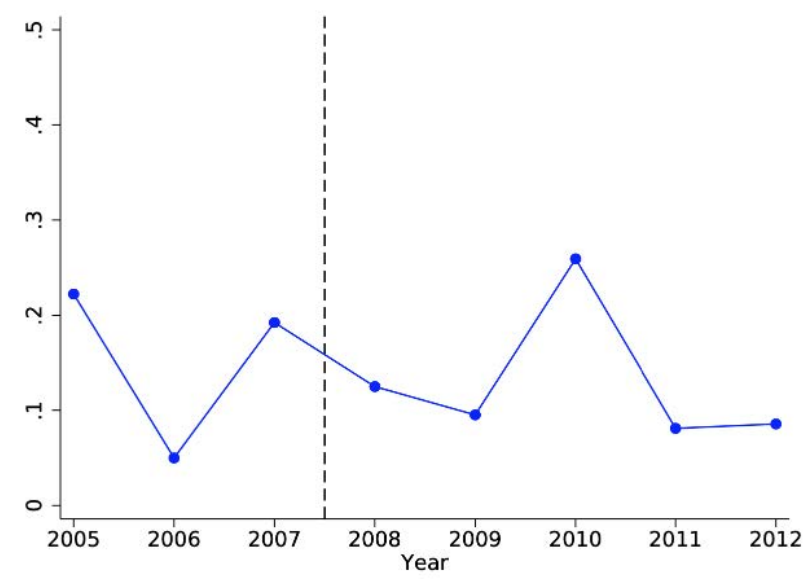

(a) Exactly zero ASC use

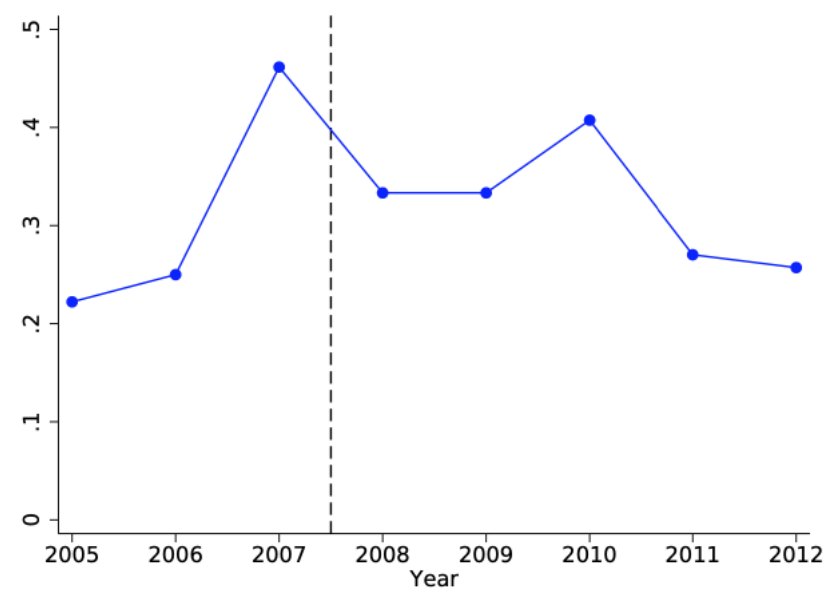

(b) $<5 \%$ ASC use

Notes: Panel (a) outcome is defined as share with precisely zero ASC cases for LCs. Panel (b) relaxes the threshold to $<5 \%$ ASC use for LC procedures among enrollees. 